INSTITUT NATIONAL DE RECHERCHE EN INFORMATIQUE ET EN AUTOMATIQUE

\title{
The Perfectly Matched Layer in Curvilinear Coordinates
}

Francis Collino et Peter Monk

\section{$\mathbf{N}^{\circ} 3049$}

Novembre 1996

THÈME 4 



\title{
The Perfectly Matched Layer in Curvilinear Coordinates
}

\author{
Francis Collino*et Peter Monk ${ }^{\dagger}$ \\ Thème 4 - Simulation et optimisation \\ de systèmes complexes \\ Projet Ondes
}

Rapport de recherche $\mathrm{n}^{\circ} 3049$ - Novembre 1996 - 49 pages

\begin{abstract}
In 1994 Bérenger showed how to construct a perfectly matched absorbing layer for the Maxwell system in rectilinear coordinates. This layer absorbs waves of any wave-length and any frequency without reflection and thus can be used to artificially terminate the domain of scattering calculations. In this paper we show how to derive and implement the Bérenger layer in curvilinear coordinates (in two space dimensions). We prove that an infinite layer of this type can be used to solve time harmonic scattering problems. We also show that the truncated Bérenger problem has a solution except at a discrete set of exceptional frequencies (which might be empty). Finally numerical results show that the curvilinear layer can produce accurate solutions in the time and frequency domain.
\end{abstract}

Key-words: Perfectly Matched Layer, computational electromagnetics, Absorbing layers

(Résumé : tsvp)

Research funded in part by a grant from AFOSR, USA. This paper has been submited to SIAM Scientific Computing

* Inria, Cerfacs

$\dagger$ Department of Mathematical Sciences, University of Delaware 


\section{Couches parfaitement adaptées en coordonnées curvilignes}

Résumé : En 1994 Bérenger a construit un modèle de couches parfaitement adaptees pour le système de Maxwell en coordonnées cartésiennes. Une telle couche absorbe les ondes sans générer de réflexion, et cela quels que soient l'angle de propagation et la fréquence. Elle peut donc être utilisée pour clore un domaine de calcul de diffractions. Dans ce papier, nous montrons comment dériver et implémenter des couches de même type en coordonnées curvilignes (et en deux dimensions). Nous montrons qu'une couche infinie résoud parfaitement le problème de diffraction. Nous montrons également que le problème de Bérenger posé en domaine tronqué est bien posé, sauf et éventuellement pour un ensemble discret de valeurs de la fréquence. Finalement, des exemples numériques montrent que ces modèles de couches en coordonnées curvilignes produisent des résultats précis.

Mots-clé : Couches absorbantes, Calcul d'ondes électromagnétiques, couches parfaitement adaptées 


\section{Abstract}

In 1994 Bérenger showed how to construct a perfectly matched absorbing layer for the Maxwell system in rectilinear coordinates. This layer absorbs waves of any wave-length and any frequency without reflection and thus can be used to artificially terminate the domain of scattering calculations. In this paper we show how to derive and implement the Bérenger layer in curvilinear coordinates (in two space dimensions). We prove that an infinite layer of this type can be used to solve time harmonic scattering problems. We also show that the truncated Bérenger problem has a solution except at a discrete set of exceptional frequencies (which might be empty). Finally numerical results show that the curvilinear layer can produce accurate solutions in the time and frequency domain.

\section{Introduction.}

Finite element, finite difference or finite volume discretizations of scattering problems are faced with the problem of truncating the infinite domain problem without introducing excessive error into the computed solution. Usually an artificial boundary is introduced on which a special absorbing boundary condition is used that reduces the reflection of waves incident on the artificial boundary. The key is to find boundary conditions that do not ruin the stability of the computation, are not too expensive and are acceptably accurate. It is difficult to satisfy these three competing criteria. Low order boundary conditions are usually stable, but in order to obtain sufficient accuracy the absorbing boundary must be far from the scatterer making the computation costly. Higher order conditions are more complex to implement and sometimes have stability problems (for a survey of absorbing boundary conditions see $[16,14])$.

Another approach to terminating the computational domain is to surround the scatterer by an absorbing layer. In the past such layers have had to be unacceptably large to control spurious reflections from the surface and interior of the layer (see for example [20]). However, in 1994, Bérenger [2] showed how to perturb the Maxwell system to provide a perfectly matched absorbing layer (abbreviated to PML for the rest of this paper) for electromagnetic scattering

$\mathrm{RR} \mathrm{n}^{\circ} 3049$ 
problems. His approach constructs an absorbing half-space such that waves incident from the standard Maxwell medium are not reflected from the half space independent of their frequency and direction of propagation. In the PML, the wave is absorbed and decays exponentially with distance into the layer. Thus the PML layer itself can be truncated to form a narrow absorbing slab with a low reflection for any angle of incidence. The scatterer can then be surrounded by perfectly matched slabs to truncate the scattering domain. This approach is easy to implement and is very effective. It has already been the subject of numerous of papers [18, 1, 5, 12, 11, 13, 25, 26, 23, 21, 15, 7, 4, 24, 3]. However, to our knowledge, there has been very little theoretical analysis of the method.

Of particular interest from the point of view of this paper is the paper of Chew and Wheedon [6] in which they show that the Bérenger PML can be constructed via a complex change of variables (see also [8]). We shall develop this viewpoint in the paper and show how to extend this idea to include a PML with a variable absorption coefficient (as is used in practice) and we shall show that method can be used in curvilinear coordinates (see also [8]). We shall show how the coordinate change is related to evaluating a continuation of the solution into the complex plane such that the solution becomes evanescent. This justifies the change of variables approach.

In this paper we shall show how to view the PML as a complex change of variables in the time harmonic scattering problem. Using this observation we shall construct a PML layer in cylindrical coordinates (in fact in two space dimensions) for the time dependent and time harmonic problems. For some scatterers the use of circular absorbing boundaries can improve the efficiency of the scheme by decreasing the area that must be meshed. We shall demonstrate how the construction of the PML is related to special function expansions of the solution and also provide a partial theoretical analysis of the time harmonic problem. Our methods extend to three dimensions and to ellipsoidal coordinates. These aspects will be reported in future papers. 


\section{Perfectly matched layers and change of va- riables}

As discussed in the introduction, in [2] Bérenger proposes a new absorbing layer model called the perfectly matched layer (PML), which has the astonishing property of generating no reflection at the interface between the free medium and the artificial absorbing medium.

In this section we shall show how Bérenger's PML can be viewed as a complex change of variables applied to the Maxwell system. In particular for the 2D Maxwell's equations, the design of the PML in the region $x>0$ is as follows. The propagation of a transverse electric wave is governed by $\left(\epsilon_{0}=\mu_{0}=1\right)$

$$
\left\{\begin{array}{l}
\frac{\partial H_{z}}{\partial t}=\frac{\partial E_{x}}{\partial y}-\frac{\partial E_{y}}{\partial x} \\
\frac{\partial E_{y}}{\partial t}=-\frac{\partial H_{z}}{\partial x}, \quad \frac{\partial E_{x}}{\partial t}=\frac{\partial H_{z}}{\partial y} .
\end{array}\right.
$$

The construction of an absorbing layer in the right half space (i.e. in the region $x>0$ ) is performed in two steps. First, we split the magnetic component into two parts according to

$$
H_{z}=H_{z x}+H_{z y}
$$

and rewrite equations (1) as

$$
\begin{cases}\frac{\partial H_{z y}}{\partial t}=\frac{\partial E_{x}}{\partial y}, & \frac{\partial H_{z x}}{\partial t}=-\frac{\partial E_{y}}{\partial x} \\ \frac{\partial E_{y}}{\partial t}=-\frac{\partial H_{z}}{\partial x}, & \frac{\partial E_{x}}{\partial t}=\frac{\partial H_{z}}{\partial y}\end{cases}
$$

At this stage, equations (2) and (3) are equivalent to the initial problem.

In the second step, a damping factor $\sigma(x),(\sigma(x)=0$ for $x<0)$ is introduced in each equation in those places where the normal derivative operator $\partial_{x}$ appears and the other parts of the equations remain unchanged:

$$
\left\{\begin{array}{l}
\frac{\partial H_{z y}}{\partial t}=\frac{\partial E_{x}}{\partial y}, \quad \frac{\partial H_{z x}}{\partial t}+\sigma H_{z x}=-\frac{\partial E_{y}}{\partial x} \\
\frac{\partial E_{y}}{\partial t}+\sigma E_{y}=-\frac{\partial H_{z}}{\partial x}, \quad \frac{\partial E_{x}}{\partial t}=\frac{\partial H_{z}}{\partial y}
\end{array}\right.
$$

$\mathrm{RR} \mathrm{n}^{\circ} 3049$ 
System (4) is Bérenger's PML model.

If we look for the time harmonic solutions with frequency $\omega$, we get

$$
\left\{\begin{array}{l}
-i \omega \hat{H}_{z y}=\frac{\partial \hat{E}_{x}}{\partial y}, \quad(-i \omega+\sigma(x)) \hat{H}_{z x}=-\frac{\partial \hat{E}_{y}}{\partial x} \\
(-i \omega+\sigma(x)) \hat{E}_{y}=-\frac{\partial \hat{H}_{z}}{\partial x}, \quad-i \omega \hat{E}_{x}=\frac{\partial \hat{H}_{z}}{\partial y}, \quad \hat{H}_{z}=\hat{H}_{z x}+\hat{H}_{z y}
\end{array}\right.
$$

We can rewrite this system of equations with the only unknown being $\hat{H}_{z}$. We have

$$
(i \omega)^{2}\left(\hat{H}_{z x}+\hat{H}_{z y}\right)+i \omega \frac{\partial \hat{E}_{x}}{\partial y}+\frac{(i \omega)^{2}}{-i \omega+\sigma(x)} \frac{\partial \hat{E}_{y}}{\partial x}=0
$$

or

$$
\omega^{2} \hat{H}_{z}+\frac{\partial^{2} \hat{H}_{z}}{\partial y^{2}}+\frac{i \omega}{i \omega-\sigma(x)} \frac{\partial}{\partial x}\left(\frac{i \omega}{i \omega-\sigma(x)} \frac{\partial \hat{H}_{z}}{\partial x}\right)=0
$$

In the region where $\sigma$ is zero, we recover the well known property that the magnetic field of a non-stationary 2D transverse electric wave satisfies the Helmholtz equation. Inside the layer, we remark that the PML model consists in making the simple substitution

$$
\frac{\partial}{\partial x} \longrightarrow \frac{\partial}{\partial x^{\prime}}=\frac{i \omega}{i \omega-\sigma(x)} \frac{\partial}{\partial x} . \quad x^{\prime}=x+\frac{i}{\omega} \int_{0}^{x} \sigma(\xi) d \xi
$$

in the initial equation, whereas the tangential derivatives are left unchanged.

Equation (6) can be easily solved (even if $\sigma$ is not constant), we get

$$
\left\{\begin{array}{l}
\hat{H}_{z}(x, y)=\frac{1}{2 \pi} \int_{-\infty}^{+\infty} H_{z}^{0}\left(k_{y}\right) e^{i k_{y} y+i k_{x} \int_{0}^{x}\left(1+\frac{i \sigma(\xi)}{\omega}\right) d \xi} d k_{y} \\
k_{x}=\omega\left[1-\frac{k_{y}^{2}}{\omega^{2}}\right]^{1 / 2}, \quad H_{z}^{0}\left(k_{y}\right)=\int_{-\infty}^{+\infty} \hat{H}_{z}(x=0, y) e^{-i k_{y} y} d y
\end{array}\right.
$$

The solutions appears as a superposition of right-going evanescent plane waves. The waves propagate without any reflection even if the variations of $\sigma$ are strong. In particular, at the interface $x=0$, no reflection occurs as the wave 
penetrates the PML. After propagating a distance $\delta$ in the $y$ direction the $k_{y}$ component of the signal is damped by a factor

$$
D\left(k_{y}\right)=e^{-\left[1-\frac{k_{y}^{2}}{\omega^{2}}\right]^{1 / 2} \int_{0}^{\delta} \sigma(\xi) d \xi}
$$

for the propagating waves (i.e. $\left|k_{y}\right|<\omega$ ) and

$$
D\left(k_{y}\right)=e^{-\omega \delta\left[\frac{k_{y}^{2}}{\omega^{2}}-1\right]^{1 / 2}}
$$

for the evanescent waves (i.e. $\left|k_{y}\right|>\omega$ ).

In case of a finite PML (it is necessary to truncate the PML to obtain a problem that can be solved numerically), a Dirichlet boundary condition is added at the right extremity, say $x=\delta$. The solutions can now be sought as

$$
\left\{\begin{array}{c}
\hat{H}_{z}(x, y)=\frac{1}{2 \pi} \int_{-\infty}^{+\infty} H_{z}^{0}\left(k_{y}\right)\left(1+R\left(k_{y}\right)\right) F\left(x, k_{y}\right) e^{i k_{y} y} d k_{y}, \\
F\left(x, k_{y}\right)=\frac{e^{i k_{x} \int_{x}^{\delta}\left(1+i \frac{\sigma(\xi)}{\omega}\right) d \xi}-e^{-i k_{x} \int_{x}^{\delta}\left(1+i \frac{\sigma(\xi)}{\omega}\right) d \xi}}{e^{i k_{x} \int_{0}^{\delta}\left(1+i \frac{\sigma(\xi)}{\omega}\right) d \xi}-e^{-i k_{x} \int_{0}^{\delta}\left(1+i \frac{\sigma(\xi)}{\omega}\right) d \xi}}
\end{array}\right.
$$

for $x>0$ and

$$
\hat{H}_{z}(x, y)=\frac{1}{2 \pi} \int_{-\infty}^{+\infty} H_{z}^{0}\left(k_{y}\right)\left(e^{i k_{x} x}+R\left(k_{y}\right) e^{-i k_{x} x}\right) e^{i k_{y} y} d k_{y}
$$

for $x<0$ located above the support of the initial conditions. These solutions are obtained by combination of plane waves which vanish at $x=\delta$ and are continuous at $x=0$. The expression of the coefficient of reflection $R\left(k_{y}\right)$ is obtained by writing down the continuity of the tangential component of the electric field

$$
\partial_{x}\left(\hat{H}_{z}\right)\left(0^{-}\right)=i \omega\left(\hat{E}_{y}\right)\left(0^{-}\right)=i \omega\left(\hat{E}_{y}\right)\left(0^{+}\right)=\frac{\omega}{\omega+i \sigma\left(0^{+}\right)} \partial_{x}\left(\hat{H}_{z}\right)\left(0^{+}\right)
$$

which gives

$$
i k_{x}(1-R)=\frac{\omega}{\omega+i \sigma\left(0^{+}\right)}\left(i k_{x}\left(1+i \frac{\sigma\left(0^{+}\right)}{\omega}\right)(1+R) \frac{1+e^{-2 i k_{x} \int_{0}^{\delta}\left(1+i \frac{i \sigma(\xi)}{\omega} d \xi\right.}}{1-e^{-2 i k_{x} \int_{0}^{\delta}\left(1+i \frac{\sigma(\xi)}{\omega} d \xi\right.}}\right.
$$

$\mathrm{RR} \mathrm{n}^{\circ} 3049$ 
or

$$
R=-e^{-2 i k_{x} \int_{0}^{\delta}\left(1+i \frac{\sigma(\xi)}{\omega}\right) d \xi}
$$

We recover Bérenger's result for the expression of the coefficient of reflection induced by a PML of width $\delta$. A direct inspection of this formula would lead us to pick $\sigma$ to be the largest possible which would allow us to obtain a reflection as weak as desired. However, this nice property is no longer true when a discretization scheme for solving numerically the equations is used. As the matter of fact, see [8], the discretization of the equations makes the layers slightly imperfectly matched. The numerical dispersion induces a reflection at the interface which is an increasing function of $\sigma \Delta x$. Thus, $\sigma$ has to be chosen small enough to control this numerical reflection coefficient and large enough to obtain a low reflection coefficient (due to the Dirichlet condition on the wall at $x=\delta$ ).

In square domains, Bérenger's PML for 2D transverse electric waves is constructed with the help of two damping functions $\sigma_{x}(x)$ and $\sigma_{y}(y)$ satisfying

$$
\begin{aligned}
& \sigma_{s}(t)=0, \text { for }|t|<\ell \\
& \sigma_{s}(t)=\sigma(t), \text { for } \quad|s|>\ell \quad \text { where } s=x, y .
\end{aligned}
$$

Equation (4) is replaced by

$$
\begin{cases}\frac{\partial H_{z y}}{\partial t}+\sigma_{y}(y) H_{z y}=\frac{\partial E_{x}}{\partial y}, & \frac{\partial H_{z x}}{\partial t}+\sigma_{x}(x) H_{z x}=-\frac{\partial E_{y}}{\partial x} \\ \frac{\partial E_{y}}{\partial t}+\sigma_{x}(x) E_{y}=-\frac{\partial H_{z}}{\partial x}, & \frac{\partial E_{x}}{\partial t}+\sigma_{y}(y) E_{x}=\frac{\partial H_{z}}{\partial y} .\end{cases}
$$

Following the same developments as above, we obtain the stationary solution as the solution of

$$
\omega^{2} \hat{H}_{z}+\frac{i \omega}{i \omega-\sigma_{y}} \frac{\partial}{\partial y}\left(\frac{i \omega}{i \omega-\sigma_{y}} \frac{\partial \hat{H}_{z}}{\partial y}\right)+\frac{i \omega}{i \omega-\sigma_{x}} \frac{\partial}{\partial x}\left(\frac{i \omega}{i \omega-\sigma_{x}} \frac{\partial \hat{H}_{z}}{\partial x}\right)=0
$$

A direct inspection shows that the Green's function for this equation is given by

$$
\hat{H}_{z}(x, y)=\frac{i}{4} H_{0}^{(1)}\left(\omega \sqrt{\tilde{x}^{2}+\tilde{y}^{2}}\right)
$$


where

$$
\tilde{x}=x+\frac{i}{\omega} \int_{0}^{x} \sigma_{x}(s) d s, \quad \tilde{y}=y+\frac{i}{\omega} \int_{0}^{y} \sigma_{y}(s) d s
$$

This is one key to understanding the nice properties of the PML model. This model is simply obtained using a complex change in variables applied to the original equation. The axes $x, y \in R$ are moved into two paths in the upper complex plane. The existence of an analytical extension of the Green's function in this upper complex plane allows us to properly define $\hat{H}_{z}$. The exponential decay of this extension ensures the damping effect of this transformation on the solution.

We end up this section by giving a variational formulation for the harmonic Bérenger's PML model with Dirichlet boundary conditions. The problem is posed in $D_{L}=(]-L, L[\times]-L, L[) \backslash \bar{\Omega}$ where $\Omega$ is a perfectly conducting scatterer entirely contained in (]$-L, L[\times]-L, L[)$ and $L>\ell$. The problem is to find $\hat{H}_{z}$ such that

$$
\left\{\begin{array}{l}
d_{x} d_{y} \hat{H}_{z}+d_{y} \frac{\partial}{\partial_{x}}\left(\frac{1}{d_{x}} \frac{\partial \hat{H}_{z}}{\partial_{x}}\right)+d_{x} \frac{\partial}{\partial_{y}}\left(\frac{1}{d_{y}} \frac{\partial \hat{H}_{z}}{\partial_{y}}\right)=0 \quad(x, y) \in D_{L} \\
\hat{H}_{z}(s)=0, \quad s \in \partial(]-L, L[\times]-L, L[) \\
\frac{\partial \hat{H}_{z}}{\partial \nu}=-g \quad s \in \partial \Omega
\end{array}\right.
$$

where $g$ is a given function and

$$
d_{x}(x)=1+\frac{i}{\omega} \sigma_{x}(x), \quad d_{y}(y)=1+\frac{i}{\omega} \sigma_{y}(y) .
$$

If

$$
X=\left\{v \in H^{1}\left(D_{L}\right) \mid v=0 \text { on } \partial(]-L, L[\times]-L, L[)\right\}
$$

the variational formulation of the above problem is simply

$$
\left\{\begin{array}{l}
\text { find } \hat{H}_{z} \in X \text { such that } \forall F \in X \\
\int_{\Omega} d_{x} d_{y} \hat{H}_{z} F d A-\int_{\Omega}\left(\frac{d_{y}}{d_{x}} \frac{\partial \hat{H}_{z}}{\partial x} \frac{\partial F}{\partial x}\right) d A+\int_{\Omega}\left(\frac{d_{x}}{d_{y}} \frac{\partial \hat{H}_{z}}{\partial y} \frac{\partial F}{\partial y}\right) d A=\int_{\partial \Omega} g F d s
\end{array}\right.
$$

$\mathrm{RR} \quad \mathrm{n}^{\circ} 3049$ 
Francis Collino et Peter Monk

This formulation can be discretized by a finite element method in the usual way way. Concerning the existence and uniqueness of the solution, a result similar to those we will obtain for Bérenger's PML in curvilinear coordinates (see Theorems 1 and 2), can be obtained.

In conclusion of this preliminary section, we have shown that the PML model can be interpreted by a change in variables and that the corresponding model can be put in a variational form suitable for a finite element method. With this in mind, we can now begin our study of a PML model in curvilinear coordinates.

\section{The time-harmonic scattering problem}

In this paper we will develop a curvilinear PML for a simple electromagnetic scattering problem using the change of variable technique introduced in the previous section. Let $\Omega$ be a smooth bounded domain in the plane, and assume that the boundary of $\Omega$ is a perfectly conducting scatter. The problem we shall consider is to compute the transverse electromagnetic field scattered from the boundary of $\Omega$. We shall start with the frequency domain problem. Let $\hat{H}$ denote the magnetic field scattered by $\partial \Omega$ then $\hat{H}$ satisfies the Helmholtz equation in the exterior of $\Omega$ :

$$
\Delta \hat{H}+k^{2} \hat{H}=0 \text { in } \mathbf{R}^{2} \backslash \bar{\Omega},
$$

where the wave number $k=\omega \sqrt{\epsilon \mu}, \omega$ is the frequency of the incident field, $\epsilon$ is the dielectric constant and $\mu$ the permeability of the surrounding medium.

On the perfectly conducting boundary, Neumann boundary data is given:

$$
\frac{\partial \hat{H}}{\partial \nu}=g \text { on } \partial \Omega
$$

where $\nu$ is the unit outward normal to $\Omega$ and $g$ is a given smooth function derived from the incoming or incident field. Finally the scattered field must satisfy the Sommerfeld radiation condition uniformly in all directions:

$$
\frac{\partial \hat{H}}{\partial \rho}-i k \hat{H}=o\left(\frac{1}{\rho^{1 / 2}}\right)
$$


where $\rho=|\boldsymbol{x}|$ is the radial coordinate. For sufficiently smooth $g$ and $\Omega$, this problem has a unique solution $H \in C^{2}\left(\mathbf{R}^{2} \backslash \bar{\Omega}\right) \cap C^{1}\left(\mathbf{R}^{2} \backslash \Omega\right.$ ) (see for example $[9])$.

Let us assume that $\bar{\Omega}$ is contained in the interior of the circle of radius $a^{\prime}$. Then for $\rho=|\boldsymbol{x}| \geq a^{\prime}$ the solution $H$ can be written using separation of variables:

$$
\hat{H}(\boldsymbol{x})=\sum_{n=-\infty}^{\infty} a_{n} H_{n}^{(1)}(k \rho) \exp (i n \theta)
$$

where $H_{n}^{(1)}$ is the Hankel function of first kind and order zero, and $\boldsymbol{x}=$ $\rho \exp (i \theta)$. This series converges uniformly for $r \geq a^{\prime}[9]$.

To obtain the Bérenger PML layer in cylindrical coordinates, we want to extend the solution given by (20) to the complex plane such that the wave becomes evanescent as the imaginary part tends to infinity. Suppose $\tilde{\rho}=$ $\tilde{\rho}_{r}+i \tilde{\rho}_{i}$ where $\tilde{\rho}_{r}$ and $\tilde{\rho}_{i}$ are real. The desired asymptotic behavior of the Hankel functions is

$$
\left|H_{m}^{(1)}(k \tilde{\rho})\right| \sim\left|\frac{C_{m}}{\sqrt{i k \tilde{\rho}}} \exp \left(i \omega\left(\tilde{\rho}_{r}+i \tilde{\rho}_{i}\right)\right)\right|=\frac{\left|C_{m}\right|}{\sqrt{k|\tilde{\rho}|}} \exp \left(-k \tilde{\rho}_{i}\right),|\tilde{\rho}| \rightarrow \infty .
$$

This implies that the correct half-plane is

$$
\Im m(k \tilde{\rho})>0 .
$$

With this observation in mind, we define the complex radius $\tilde{\rho}$. We choose $a>a^{\prime}$ and a real (artificial) absorption coefficient $\sigma=\sigma(\rho)$ parametrized by $\rho$ satisfying $\sigma(\rho)=0$ for $\rho<a$ and $\sigma(\rho)>0$ for $\rho>a$, then

$$
\tilde{\rho}= \begin{cases}\rho+\int_{a}^{\rho} \frac{i \sigma(s)}{\omega} d s & \text { if } \rho \geq a \\ \rho & \text { if } a^{\prime}<\rho<a\end{cases}
$$

We also assume that

$$
\lim _{\rho \rightarrow \infty} \int_{a}^{\rho} \sigma(s) d s=\infty
$$

Note that

$$
\frac{d \tilde{\rho}}{d \rho}=d= \begin{cases}1+\frac{i \sigma(\rho)}{\omega} & \text { if } \rho \geq a \\ 1 & \text { if } a^{\prime}<\rho<a\end{cases}
$$

$\mathrm{RR} \mathrm{n}^{\circ} 3049$ 
It will prove convenient to define $\bar{\sigma}$ by

$$
\bar{\sigma}= \begin{cases}\frac{1}{\rho} \int_{a}^{\rho} \sigma(s) d s & \text { if } \rho \geq a \\ 0 & \text { if } \rho<a\end{cases}
$$

Then $\tilde{\rho}$ and $\rho$ are related by $\bar{d}$ as follows

$$
\tilde{\rho}=\rho\left(1+\frac{i \bar{\sigma}}{\omega}\right)=\rho \bar{d}
$$

Now we can define the Bérenger solution $\hat{H}^{B}$ for $\boldsymbol{x} \in \mathbf{R}^{2} \backslash \bar{\Omega}$ as follows:

$$
\hat{H}^{B}(\boldsymbol{x})= \begin{cases}\hat{H}(\boldsymbol{x}) & \text { if }|\boldsymbol{x}| \leq a^{\prime} \\ \sum_{n=-\infty}^{\infty} a_{n} H_{n}^{(1)}(k \tilde{\rho}) \exp (i n \theta) & \text { if }|\boldsymbol{x}|>a^{\prime}\end{cases}
$$

We need to derive a boundary value problem satisfied by $\hat{H}^{B}$. If $\boldsymbol{x} \in \mathbf{R}^{2} \backslash \bar{\Omega}$ and $|\boldsymbol{x}|<a^{\prime}, \hat{H}^{B}$ satisfies the standard Helmholtz equation. Across the circle $|\boldsymbol{x}|=a^{\prime}$ the function $\hat{H}^{B}$ and it's normal derivative are continuous. Thus it remains to derive an equation in $|\boldsymbol{x}|>a^{\prime}$. In this region $\hat{H}^{B}$ is a sum of Hankel functions and hence satisfies the Helmholtz equation (17) but using $\tilde{\rho}$ in place of $\rho$

$$
-k^{2} \hat{H}^{B}=\frac{1}{\tilde{\rho}}\left(\frac{\partial}{\partial \tilde{\rho}}\left(\tilde{\rho} \frac{\partial}{\partial \tilde{\rho}} \hat{H}^{B}\right)+\frac{1}{\tilde{\rho}} \frac{\partial^{2}}{\partial \theta^{2}} \hat{H}^{B}\right) .
$$

We can start by using the chain rule:

$$
\frac{\partial H}{\partial \tilde{\rho}}=\frac{\partial H}{\partial \rho} \frac{\partial \rho}{\partial \tilde{\rho}}=\frac{1}{d} \frac{\partial H}{\partial \rho}
$$

Hence using this result and (21) we can rewrite (23) in terms of $\rho$. We obtain:

$$
-k^{2} \hat{H}^{B}=\frac{1}{\bar{d}} \frac{1}{\rho}\left(\frac{1}{d} \frac{\partial}{\partial \rho}\left(\frac{\bar{d} \rho}{d} \frac{\partial}{\partial \rho} \hat{H}^{B}\right)+\frac{1}{\bar{d} \rho} \frac{\partial^{2}}{\partial \theta^{2}} \hat{H}^{B}\right)
$$

which we rewrite slightly as

$$
-k^{2} d \bar{d} \hat{H}^{B}=\frac{1}{\rho}\left(\frac{\partial}{\partial \rho}\left(\frac{\bar{d} \rho}{d} \frac{\partial}{\partial \rho} \hat{H}^{B}\right)+\frac{d}{\bar{d} \rho} \frac{\partial^{2}}{\partial \theta^{2}} \hat{H}^{B}\right)
$$


It is convenient (from the point of view of implementation in a standard finite element code) to transform this equation back to rectangular coordinates to obtain

$$
-k^{2} d \bar{d} \hat{H}^{B}=\nabla \cdot\left(A \nabla \hat{H}^{B}\right)
$$

where $A$ is the following matrix function:

$$
A(\rho, \theta)=\left(\begin{array}{cc}
\frac{\bar{d}}{d} \cos ^{2}(\theta)+\frac{d}{\bar{d}} \sin ^{2}(\theta) & \cos (\theta) \sin (\theta)\left(\frac{\bar{d}}{d}-\frac{d}{\bar{d}}\right) \\
\cos (\theta) \sin (\theta)\left(\frac{\bar{d}}{d}-\frac{d}{\bar{d}}\right) & \overline{\frac{d}{d}} \sin ^{2}(\theta)+\frac{d}{\bar{d}} \cos ^{2}(\theta)
\end{array}\right)
$$

If $\rho \leq a^{\prime}$ we define $A=I$ (the identity matrix), and $d=\bar{d}=1$ The problem of computing the Bérenger solution $\hat{H}^{B}$ is thus the problem of finding $\hat{H}^{B}$ such that

$$
\begin{aligned}
\nabla \cdot\left(A \nabla \hat{H}^{B}\right)+k^{2} d \bar{d} \hat{H}^{B} & =0 \text { in } \mathbf{R}^{2} \backslash \bar{\Omega} \\
\frac{\partial \hat{H}^{B}}{\partial \nu} & =g \text { on } \partial \Omega \\
\left|\hat{H}^{B}\right| & \text { is uniformly bounded as }|\rho| \rightarrow \infty
\end{aligned}
$$

Here the boundedness assumption has replaced the standard Sommerfeld condition at infinity (because the solution is assumed to be decaying at infinity). We can now state and prove our first theorem:

Theorem 1 There exists a unique solution of the Bérenger problem (26)-(28) given by (22).

Remark. This theorem shows that the solution of the curvilinear Bérenger time-harmonic scattering problem is the desired physical solution of the standard scattering problem in the neighborhood of the scatterer.

Proof: Existence is not a problem since we have constructed the boundary value problem (26)-(28) so that (22) (derived from the known solution of the standard scattering problem) is a solution.

To prove uniqueness we can modify the standard proof as follows. We set $g=0$. Let $D_{a^{\prime}}=\left\{\boldsymbol{x} \in \mathbf{R}^{2} \backslash \bar{\Omega}|| \boldsymbol{x} \mid<a^{\prime}\right\}$ then using the fact that (26) is just the Helmholtz equation in $D_{a^{\prime}}$ we can use Green's theorem applied to $D_{a^{\prime}}$ to show that if $g=0$ then

$$
\int_{|\boldsymbol{x}|=a^{\prime}} \overline{\hat{H}^{B}} \frac{\partial \hat{H}^{B}}{\partial r}-\hat{H}^{B} \frac{\partial \overline{\hat{H}^{B}}}{\partial r} d s=0 .
$$

$\mathrm{RR} \mathrm{n}^{\circ} 3049$ 
But for $r>a^{\prime}, \hat{H}^{B}$ satisfies (25) and hence is a sum of Hankel functions:

$$
\hat{H}^{B}=\sum_{n=-\infty}^{\infty}\left(a_{n} H_{n}^{(1)}(k \tilde{\rho})+b_{n} H_{n}^{(2)}(k \tilde{\rho})\right) \exp (i n \theta) .
$$

Since we assume that $\sigma$ is chosen so that $\Im(k \tilde{\rho}) \rightarrow \infty$ as $\rho \rightarrow \infty$ the large argument asymptotics of the Hankel functions (note $\Re(k \tilde{\rho})=\rho$ ) shows that $H_{n}^{(2)}(k \tilde{\rho}),-\infty<n<\infty$ is unbounded as $\rho \rightarrow \infty$. Hence to satisfy the radiation condition (28) we must require $b_{n}=0-\infty<n<\infty$. Now using the Hankel function expansion in (29) shows that

$$
k \sum_{n=-\infty}^{\infty}\left|a_{n}\right|^{2}\left(H_{n}^{(1)}\left(k a^{\prime}\right) \overline{H_{n}^{(1) \prime}\left(k a^{\prime}\right)}-\overline{H_{n}^{(1)}\left(k a^{\prime}\right)} H_{n}^{(1) \prime}\left(k a^{\prime}\right)\right)=0
$$

where we have used the fact that $\tilde{\rho}=\rho$ on $a^{\prime} \leq \rho \leq a$. Using the non-vanishing of the Wronskian of the two Hankel functions we conclude that $a_{n}=0$ for all $n$. Hence $\hat{H}^{B}(\boldsymbol{x})=0$ for $|\boldsymbol{x}| \geq a^{\prime}$, and a standard continuation argument shows that $\hat{H}^{B}=0$ in $\mathbf{R}^{2} \backslash \bar{\Omega}$ (see for example [9]). This completes the proof.

The boundary value problem (26)-(28) is still not suitable for computation since it is posed on an infinite domain. However truncation of this problem is not as delicate as for the standard scattering problem since the solution decays exponentially outside the circle $\rho=a$. For example we can impose the Dirichlet boundary condition on a suitably large radius circle containing the scatter (in three dimensions this would be equivalent to specifying that the tangential component of the magnetic field vanishes on the artificial boundary),

Let

$$
D_{b}=\left\{\boldsymbol{x} \in \mathbf{R}^{2} \backslash \bar{\Omega}|| \boldsymbol{x} \mid<b\right\}
$$

On the outer boundary we impose the Dirichlet boundary condition

$$
\hat{H}^{B}=0 \text { on }|\boldsymbol{x}|=b .
$$

Then the first boundary value problem we shall approximate numerically is to find $\hat{H}^{B}$ such that (26) is satisfied in $D_{b}$ with boundary data given by $(27)$ on $\partial \Omega$ and by (30) on the artificial boundary $|\boldsymbol{x}|=b$.

As we shall see, better numerical behavior can be obtained if we use an absorbing boundary condition on the outer boundary. So to further improve 
the efficiency of our numerical experiments we use a simple absorbing boundary condition on the artificial boundary. We impose the Sommerfeld radiation condition suitable for the evanescent Hankel function:

$$
\frac{\partial \hat{H}^{B}}{\partial \rho}-i k d \hat{H}^{B}=0 \text { on }|\boldsymbol{x}|=b .
$$

Then the field $\hat{H}^{B}$ satisfies the Robin problem (26), (27) and (31).

The obvious questions are whether the problems outlined above have unique solutions and whether their solution is close to the solution of the infinite domain Bérenger problem (in particular we want to know if the solution is close to the true scattering solution close to $\Omega$ ). In particular, does the absorbing boundary condition improve the method. We can provide a partial answer to the first of these questions as the following theorem shows but we have been unable to prove the approximation property.

Before stating the theorem we define the constant $M>0$ by

$$
M=\sup _{\rho \in(a, b)} \sigma(\rho)+\bar{\sigma}(\rho)
$$

We will prove our theorem by allowing complex valued $\omega$ in the following set $\mathcal{U} \subset \mathbf{C}$ which contains the positive real axis with the exception of $\omega=0$ (where physically interesting values of $\omega$ lie):

$$
\mathcal{U}=\{\omega \in \mathbf{C}, \Im(\omega)>0\} \cup\left\{\omega=\omega_{r}+i \omega_{i}, \omega_{r} \neq 0,\left|\omega_{i}\right|<\omega_{r}^{2} / M\right\} .
$$

Recall that $k=\omega \sqrt{\epsilon \mu}$.

Theorem 2 Suppose $g \in H^{-1 / 2}(\partial \Omega)$. Then:

1. The boundary value problem (26), (27) and (30) on $D_{b}$ has a unique solution $\hat{H}^{B}$ in $H^{1}\left(D_{b}\right)$ for every real $k$ except possibly for a discrete set of values of $k$.

2. The same conclusion holds if $\hat{H}^{B} \in H^{1}\left(D_{b}\right)$ satisfies the Robin problem (26), (27) and (31). 
Remark. We have been unable to rule out the possibility that a unique solution fails to exist for some real $k$.

Proof: The proof of the two conclusions of the theorem are almost identical, so we will prove only the case of the Robin problem which is more interesting in practice.

The proof rests on the analytic Fredholm theorem (see [10]). First we pose the Bérenger problem (26), (27) and (31) as a weak problem on the space $H^{1}\left(D_{b}\right)$ (for the Dirichlet problem we would use the subspace $X=\{u \in$ $H^{1}\left(D_{b}\right) \mid u=0$ on $\left.\left.|\boldsymbol{x}|=b\right\}\right)$. Let

$$
(u, v)=\int_{D_{b}} u v d A, \quad<u, v>_{\partial \Omega}=\int_{\partial \Omega} u v d s \text { and }<u, v>_{b}=\int_{|\boldsymbol{x}|=b} u v d s
$$

then we seek $\hat{H}^{B} \in H^{1}\left(D_{b}\right)$ such that

$$
\left(A \nabla \hat{H}^{B}, \nabla v\right)-k^{2}(d \bar{d} u, v)-i k<\bar{d} u, v>_{b}=-<g, v>_{\partial \Omega} \forall v \in H^{1}\left(D_{b}\right) .
$$

Now we define two operators $\mathcal{A}(\omega): H^{1}\left(D_{b}\right) \rightarrow H^{1}\left(D_{b}\right)$ and $\mathcal{B}(\omega): H^{1}\left(D_{b}\right) \rightarrow$ $H^{1}\left(D_{b}\right)$ by

$$
\begin{aligned}
(\nabla \mathcal{A} u, \nabla v)+(\mathcal{A} u, v) & =(A \nabla u, \nabla v)+(u, v) \\
(\nabla \mathcal{B} u, \nabla v)+(\mathcal{B} u, v) & =\left(\left(k^{2} d \bar{d}+1\right) u, v\right)+i k<\bar{d} u, v>_{b}
\end{aligned}
$$

for all $u, v \in H^{1}\left(D_{b}\right)$. Both operators are well defined by the Riez representation theorem. Note that because $d, \bar{d}$ and $k$ depend on $\omega$, the operators $\mathcal{A}$ and $\mathcal{B}$ depend on $\omega$.

Since the coefficients defining $A$ are analytic at each $\omega \in \mathcal{U}$, the operator $\mathcal{A}$ is analytic in $\omega$. Next we show that $\mathcal{A}^{-1}$ exists and is continuous by using the Lax-Milgram lemma. The continuity of $\mathcal{A}$ is obvious so it remains to show that $\mathcal{A}$ is strongly coercive. But

$$
|(u, \bar{u})+(A \nabla u \nabla \bar{u})|=|(\nabla \mathcal{A} u, \nabla \bar{u})+(\mathcal{A} u, \bar{u})| \leq C|| \mathcal{A} u\left\|_{H^{1}\left(D_{b}\right)}|| u\right\|_{H^{1}\left(D_{b}\right)}
$$

and so strong coercivity is proved if there is a positive constant $\delta$ (possibly depending on $\omega$ ) such that

$$
|(u, \bar{u})+(A \nabla u \nabla \bar{u})| \geq\left.\delta|| u\right|_{H^{1}\left(D_{b}\right)} ^{2} .
$$


For any $u \in H^{1}\left(D_{b}\right)$

$$
|(A \nabla u, \nabla \bar{u})| \geq \Re((A \nabla u, \nabla u))=\int_{\boldsymbol{x}_{\in} \in D_{b},|\boldsymbol{x}|<a^{\prime}}|\nabla u|^{2} d A+\int_{a^{\prime}<|\boldsymbol{x}|<b} \Re(\nabla u \cdot A \nabla \bar{u}) d A .
$$

But from the definition of the Bérenger layer:

$$
\int_{a^{\prime}<|\boldsymbol{x}|<b} \Re(\nabla u \cdot A \nabla \bar{u}) d A .=\int_{a^{\prime}}^{b} \int_{0}^{2 \pi} \rho\left(\Re\left(\frac{\bar{d}}{d}\right)\left|\frac{\partial u}{\partial \rho}\right|^{2}+\Re\left(\frac{d}{\bar{d} \rho^{2}}\right)\left|\frac{\partial u}{\partial \theta}\right|^{2}\right) d \theta d r .
$$

It thus suffices to show that

$$
\min (\Re(d / \bar{d}), \Re(\bar{d} / d)) \geq \delta>0
$$

where $\delta$ is independent of $\rho$ but can depend on $\omega$.

We shall prove the above inequality for $\Re(d / \bar{d})$, the other inequality being similar. Note that if $\omega=\omega_{r}+i \omega_{i}$ then

$$
\Re\left(\frac{d}{\bar{d}}\right)=\frac{\omega_{r}^{2}+\left(\sigma+\omega_{i}\right)\left(\bar{\sigma}+\omega_{i}\right)}{\omega_{r}^{2}+\left(\bar{\sigma}+\omega_{i}\right)^{2}} .
$$

Hence if $\omega_{i}>0$ then $\Re(d / \bar{d})$ is a monotonic function of $\omega_{r}$ and so

$\Re\left(\frac{d}{\bar{d}}\right) \geq \min \left(1, \frac{\sigma+\omega_{i}}{\bar{\sigma}+\omega_{i}}\right) \geq \min \left(1, \frac{\omega_{i}}{\bar{\sigma}+\omega_{i}}\right) \geq \min \left(1, \frac{\omega_{i}}{M+\omega_{i}}\right)=\delta_{1}(\omega)>0$.

But if $\omega_{r} \neq 0$ and $\left|\omega_{i}\right|<\omega_{r}^{2} / M$ then

$$
\Re\left(\frac{d}{\bar{d}}\right) \geq \frac{\omega_{r}^{2}+(\sigma+\bar{\sigma}) \omega_{i}}{\omega_{r}^{2}} \geq \frac{\omega_{r}^{2}-M \omega_{i}}{\omega_{r}^{2}}=\delta_{2}(\omega)>0 .
$$

Hence $\Re(d / \bar{d}) \geq \min \left(\delta_{1}, \delta_{2}\right)>0$. Having proved the strong coercivity of $\mathrm{A}$, we can now conclude that $\mathcal{A}$ is invertible for any $\omega \in \mathcal{U}$.

The operator $\mathcal{B}$ is compact, because if $w=\mathcal{B} u$ then $w \in H^{1}\left(D_{b}\right)$ satisfies the following boundary value problem:

$$
\begin{aligned}
-\Delta w+w & =k^{2} d \bar{d} u \text { in } D_{b} \\
\frac{\partial w}{\partial \nu} & =0 \text { on } \partial \Omega \\
\frac{\partial w}{\partial r} & =-i k \bar{d} u \text { on }|\boldsymbol{x}|=b
\end{aligned}
$$

$\mathrm{RR} \mathrm{n}^{\circ} 3049$ 
Since $d$ and $\bar{d}$ are functions in $L_{\infty}\left(D_{b}\right)$, standard elliptic regularity theory implies that $\|w\|_{H^{2}(\Omega)} \leq C\|u\|_{H^{1}(\Omega)}$ but since $H^{2}(\Omega)$ is compactly embedded in $H^{1}(\Omega)$ we conclude that $\mathcal{B}$ is compact. Furthermore $\mathcal{B}$ is an operator valued analytic function of $k$ for all $k$.

Now let $G \in H^{1}(\Omega)$ be defined by

$$
(\nabla G, \nabla v)+(G, v)=<g, v>_{b} \quad \forall v \in H^{1}(\Omega)
$$

then the Bérenger problem is equivalent to finding $\hat{H}^{B} \in H^{1}(\Omega)$ such that

$$
\mathcal{A} \hat{H}^{B}-\mathcal{B} \hat{H}^{B}=G
$$

Since $\mathcal{A}^{-1}$ is bounded, $\mathcal{A}^{-1} \mathcal{B}$ is compact (and an operator valued analytic function of $k$ ) and so the analytic Fredholm alternative theorem is applicable. It remains to decide between the alternatives of this theorem. To do this shall prove that a solution of the Bérenger problem exists when $\omega=i$. Once this is proved, the theorem is proved since the analytic Fredholm theorem implies that $\left(I-\mathcal{A}^{-1} \mathcal{B}\right)^{-1}$ exists except possibly for a discrete set of exceptional wavenumbers.

When $\omega=i$, the functions $d$ and $\bar{d}$ are real and positive since

$$
d=(1+\sigma)>0 \text {, and } \bar{d}=(1+\bar{\sigma})>0 .
$$

Hence the matrix $A$ is real and uniformly positive definite (when $\omega=i$ ) and $d \bar{d}>0$. The weak problem is to find $\hat{H}^{B} \in H^{1}\left(D_{b}\right)$ such that

$$
\left(A \nabla \hat{H}^{B}, \nabla v\right)+\epsilon \mu(d \bar{d} u, v)+\sqrt{\epsilon \mu}<\bar{d} u, v>_{b}=-<g, v>_{\partial \Omega} \forall v \in H^{1}\left(D_{b}\right)
$$

But in this case the bilinear form associated with this problem is coercive since if $u \in H^{1}(\Omega)$ then

$$
(A \nabla u, \nabla \bar{u})+\epsilon \mu(d \bar{d} u, \bar{u})+\sqrt{\epsilon \mu}<\bar{d} u, \bar{u}>_{b} \geq C\left(\|\nabla u\|_{L_{2}\left(D_{b}\right)}^{2}+\|u\|_{L_{2}\left(D_{b}\right)}^{2}\right) .
$$

Hence the Lax-Milgram lemma shows that a unique solution exists for (34). 


\section{The Time Dependent Problem.}

Bérenger first proposed his PML for the time dependent Maxwell system. Here we describe how to generalize Bérenger's PML to allow for an annular absorbing layer in the time domain. The scattering problem is again to predict transverse mode scattering from an infinite perfectly conducting cylinder occupying the region $\Omega$ described in the previous section (although, as we have seen, the details of the scatterer are unimportant from the point of view of developing the PML equations).

We assume that at $t=0$ the fields vanish for $\boldsymbol{x} \in \mathbf{R}^{2} \backslash \bar{\Omega}$. After $t=0$, an incident wave interacts with the scatterer to produce a scattered electromagnetic field where the electric field $\boldsymbol{E}^{M}=\left(E_{1}^{M}, E_{2}^{M}\right)^{T}$ and the magnetic field $H^{M}$ satisfy the two dimensional Maxwell system

$$
\begin{aligned}
\epsilon \frac{\partial E_{1}^{M}}{\partial t} & =\frac{\partial H^{M}}{\partial y} \text { in } \mathbf{R}^{2} \backslash \bar{\Omega} \\
\epsilon \frac{\partial E_{2}^{M}}{\partial t} & =-\frac{\partial H^{M}}{\partial x} \text { in } \mathbf{R}^{2} \backslash \bar{\Omega} \\
\mu \frac{\partial H}{\partial t} & =\frac{\partial E_{1}^{M}}{\partial y}-\frac{\partial E_{2}^{M}}{\partial x} \text { in } \mathbf{R}^{2} \backslash \bar{\Omega}
\end{aligned}
$$

with zero initial data and boundary data appropriate for a perfectly conducting obstacle:

$$
\nu_{1} E_{2}^{M}-\nu_{2} E_{1}^{M}=\gamma \quad \text { on } \partial \Omega,
$$

where $\gamma$ is a given function (which is determined by the incident electromagnetic field).

As we have seen in the previous section, the curvilinear Bérenger PML can be derived by writing the frequency domain equations in curvilinear coordinates and changing variables. We start by writing the Maxwell system in cylindrical coordinates. For $a^{\prime}<\rho$ the electric field $\boldsymbol{E}^{M}=\left(E_{\rho}^{M}, E_{\theta}^{M}\right)^{T}$ and magnetic field $H^{M}$ satisfy

$$
\begin{aligned}
\epsilon \frac{\partial E_{\rho}^{M}}{\partial t} & =\frac{1}{\rho} \frac{\partial H^{M}}{\partial \theta} \\
\epsilon \frac{\partial E_{\theta}^{M}}{\partial t} & =-\frac{\partial H^{M}}{\partial \rho}
\end{aligned}
$$

$\mathrm{RR} \mathrm{n}^{\circ} 3049$ 


$$
\mu \frac{\partial H}{\partial t}=-\frac{1}{\rho}\left(\frac{\partial}{\partial \rho}\left(\rho E_{\theta}^{M}\right)-\frac{\partial E_{\rho}^{M}}{\partial \theta}\right)
$$

To derive the Bérenger medium, we move to the frequency domain. If we assume that $E_{\rho}^{M}=\hat{E}_{\rho}^{M} \exp (-i \omega t)$ and similarly for the remaining variables we obtain the time harmonic Maxwell system:

$$
\begin{aligned}
-i \omega \epsilon \hat{E}_{\rho}^{M} & =\frac{1}{\rho} \frac{\partial \hat{H}^{M}}{\partial \theta} \\
-i \omega \epsilon \hat{E}_{\theta}^{M} & =-\frac{\partial \hat{H}^{M}}{\partial \rho} \\
-i \omega \mu \hat{H}^{M} & =-\frac{1}{\rho}\left(\frac{\partial}{\partial \rho}\left(\rho \hat{E}_{\theta}^{M}\right)-\frac{\partial \hat{E}_{\rho}^{M}}{\partial \theta}\right)
\end{aligned}
$$

For the time dependent problem, we proceed analogously to the previous section. The Bérenger system is just the standard Maxwell system, written using $\tilde{\rho}$ in place of $\rho$ (the fields now carry the superscript $B$ to indicate that we are considering a Bérenger medium):

$$
\begin{aligned}
-i \omega \epsilon \hat{E}_{\rho}^{B} & =\frac{1}{\tilde{\rho}} \frac{\partial \hat{H}^{B}}{\partial \theta} \\
-i \omega \epsilon \hat{E}_{\theta}^{B} & =-\frac{\partial \hat{H}^{B}}{\partial \tilde{\rho}} \\
-i \omega \mu \hat{H}^{B} & =-\frac{1}{\tilde{\rho}}\left(\frac{\partial}{\partial \tilde{\rho}}\left(\tilde{\rho} \hat{E}_{\theta}^{B}\right)-\frac{\partial \hat{E}_{\rho}^{B}}{\partial \theta}\right)
\end{aligned}
$$

Note that if we use (35) and (36) to eliminate $\hat{E}_{\rho}^{B}$ and $\hat{E}_{\theta}^{B}$ from (37) we obtain (25). As in the case of the time harmonic problem, we want to derive the equivalent of this system using real coordinates. Using (24) implies that (36) can be written:

$$
-i \omega \epsilon \hat{E}_{\theta}^{B}+\epsilon \sigma \hat{E}_{\theta}^{B}=-\frac{\partial \hat{H}^{B}}{\partial \rho}
$$

We can easily rewrite (35) using the fact that $\tilde{\rho}=\bar{d} \rho$ to obtain:

$$
-i \omega \epsilon \hat{E}_{\rho}^{B}+\epsilon \bar{\sigma} \hat{E}_{\rho}^{B}=\frac{1}{\rho} \frac{\partial \hat{H}^{B}}{\partial \theta}
$$


It remains to rewrite (37). We define the auxiliary Bérenger variable $\hat{H}^{(\rho)}$ by

$$
-i \omega \mu \hat{H}^{(\rho)}=-\frac{1}{d} \frac{\partial}{\partial \rho} \hat{E}_{\theta}^{B}
$$

Expanding the derivative, we can rewrite (37) as

$$
-i \omega \mu \hat{H}^{B}=-\frac{1}{\tilde{\rho}}\left(\hat{E}_{\theta}^{B}+\tilde{\rho} \frac{\partial}{\partial \tilde{\rho}} \hat{E}_{\theta}^{B}-\frac{\partial \hat{E}_{\rho}^{B}}{\partial \theta}\right) .
$$

We can then change variables back to $\rho$ and add and subtract $(1 / \bar{d} \rho)(\partial / \partial \rho) \hat{E}_{\theta}^{B}$ to both sides to obtain

$$
-i \omega \mu \hat{H}^{B}=-\left(\frac{1}{d}-\frac{1}{\bar{d}}\right) \frac{\partial}{\partial \rho} \hat{E}_{\theta}^{B}-\frac{1}{\bar{d} \rho}\left(\frac{\partial}{\partial \rho}\left(\rho \hat{E}_{\theta}^{B}\right)-\frac{\partial \hat{E}_{\rho}^{B}}{\partial \theta}\right) .
$$

Hence

$$
-i \omega \mu \bar{d} \hat{H}^{B}=(d-\bar{d}) i \omega \mu \hat{H}^{(\rho)}-\frac{1}{\rho}\left(\frac{\partial}{\partial \rho}\left(\rho \hat{E}_{\theta}^{B}\right)-\frac{\partial \hat{E}_{\rho}^{B}}{\partial \theta}\right)
$$

and this becomes

$$
-i \omega \mu \hat{H}^{B}+\mu \bar{\sigma} \hat{H}^{B}+\mu(\sigma-\bar{\sigma}) \hat{H}^{(\rho)}=-\frac{1}{\rho}\left(\frac{\partial}{\partial \rho}\left(\rho \hat{E}_{\theta}^{B}\right)-\frac{\partial \hat{E}_{\rho}^{B}}{\partial \theta}\right) .
$$

Collecting the equations (38), (39), (40) and (41) we obtain the following time harmonic Bérenger system:

$$
\begin{aligned}
-i \omega \epsilon \hat{E}_{\rho}^{B}+\epsilon \bar{\sigma} \hat{E}_{\rho}^{B} & =\frac{1}{\rho} \frac{\partial \hat{H}^{B}}{\partial \theta} \\
-i \omega \epsilon \hat{E}_{\theta}^{B}+\epsilon \sigma \hat{E}_{\theta}^{B} & =-\frac{\partial \hat{H}^{B}}{\partial \rho} \\
-i \omega \mu \hat{H}^{B}+\mu \bar{\sigma} \hat{H}^{B}+\mu(\sigma-\bar{\sigma}) \hat{H}^{(\rho)} & =-\frac{1}{\rho}\left(\frac{\partial}{\partial \rho}\left(\rho \hat{E}_{\theta}^{B}\right)-\frac{\partial \hat{E}_{\rho}^{B}}{\partial \theta}\right) \\
-i \omega \mu \hat{H}^{(\rho)}+\mu \sigma \hat{H}^{(\rho)} & =-\frac{\partial}{\partial \rho} \hat{E}_{\theta}^{B}
\end{aligned}
$$

$\mathrm{RR} \mathrm{n}^{\circ} 3049$ 
Finally, we can move back to the time domain to obtain:

$$
\begin{aligned}
\epsilon \frac{\partial E_{\rho}^{B}}{\partial t}+\epsilon \bar{\sigma} E_{\rho}^{B} & =\frac{1}{\rho} \frac{\partial H^{B}}{\partial \theta} \\
\epsilon \frac{\partial E_{\theta}^{B}}{\partial t}+\epsilon \sigma E_{\theta}^{B} & =-\frac{\partial H^{B}}{\partial \rho} \\
\mu \frac{\partial H^{B}}{\partial t}+\mu \bar{\sigma} H^{B}+\mu(\sigma-\bar{\sigma}) H^{(\rho)} & =-\frac{1}{\rho}\left(\frac{\partial}{\partial \rho}\left(\rho E_{\theta}^{B}\right)-\frac{\partial E_{\rho}^{B}}{\partial \theta}\right) \\
\mu \frac{\partial H^{(\rho)}}{\partial t}+\mu \sigma H^{(\rho)} & =-\frac{\partial}{\partial \rho} E_{\theta}^{B}
\end{aligned}
$$

\section{$5 \quad$ Numerical Results}

\subsection{A Special Case of the Time Harmonic Problem}

In the special case of a concentric circular scatterer we can solve the standard scattering problem and Bérenger problem using special functions. We will use this case to examine the improvement gained by using the Robin problem over the Dirichlet problem. Let us suppose that the scatter $\Omega$ is a circle of radius $a^{\prime}$.

First let us consider the standard scattering problem of computing $\hat{H}$ such that (17), (18) and (19) are satisfied in the case when the scatterer $\Omega$ is a disk of radius $a^{\prime}$ and the boundary data is

$$
g=-\frac{\partial \exp (i k x)}{\partial \rho} \text { on }|\boldsymbol{x}|=a^{\prime}
$$

where $\boldsymbol{x}=(x, y)$. This problem has the solution

$$
\hat{H}(\rho, \theta)=\sum_{n=-\infty}^{\infty} a_{n} H_{n}^{(1)}(k \rho) \exp (i n \theta)
$$

where

$$
a_{n}=-\frac{i^{n} J_{n}^{\prime}\left(k a^{\prime}\right)}{H_{n}^{(1) \prime}(k a)}
$$


If we truncate the domain using a circle of radius $b$, use a Bérenger layer in $a^{\prime}<a \leq \rho<b$ and use the Dirichlet condition on $\rho=b$ we must solve

$$
\begin{aligned}
\nabla \cdot\left(A \nabla \hat{H}^{B}\right)+k^{2} d \bar{d} \hat{H}^{B} & =0 \text { in } D_{b} \\
\frac{\partial \hat{H}^{B}}{\partial \rho} & =\frac{\partial \exp (i k x)}{\partial \rho} \text { when } \rho=a^{\prime} \\
\hat{H}^{B} & =0 \text { when } \rho=b
\end{aligned}
$$

Let use denote by $\tilde{\rho}_{b}=\left.\tilde{\rho}\right|_{\rho=b}$, then the above problem has the solution

$$
\hat{H}^{B}(\rho, \theta)=\sum_{n=-\infty}^{\infty}\left(a_{n}^{B} H_{n}^{(1)}(k \tilde{\rho})+b_{n}^{B} H_{n}^{(2)}(k \tilde{\rho})\right) \exp (i n \theta)
$$

where

$$
a_{n}^{B}=-\frac{H_{n}^{(2)}\left(k \tilde{\rho}_{b}\right)}{H_{n}^{(1)}\left(k \tilde{\rho}_{b}\right)} b_{n}^{B} \text { and } b_{n}^{B}=-\frac{i^{n} J_{n}^{\prime}\left(k a^{\prime}\right) H_{n}^{(1)}\left(k \tilde{\rho}_{b}\right)}{W}
$$

provided $W \neq 0$ where

$$
W=H_{n}^{(2) \prime}\left(k a^{\prime}\right) H_{n}^{(1)}\left(k \tilde{\rho}_{b}\right)-H_{n}^{(1) \prime}\left(k a^{\prime}\right) H_{n}^{(2)}\left(k \tilde{\rho}_{b}\right) .
$$

When $W=0$ the problem is not uniquely solvable. Of course the solution $\hat{H}^{B}$ is independent of the details of the Bérenger layer and depends only on $\tilde{\rho}_{b}$. This is not the case when numerical methods are used to discretize the Bérenger layer since there will be spurious reflections from within the layer and the layer must be designed to minimize these (as well as reach the desired value of $\left.\tilde{\rho}_{b}\right)$.

We can evaluate the error in the Bérenger solution on the circle $\rho=a^{\prime}$ by computing the relative error:

$$
\left\|\hat{H}-\hat{H}^{B}\right\|_{R}=\frac{\sum_{n=-\infty}^{\infty}\left|\left(a_{n}-a_{n}^{B}\right) H_{n}^{(1)}\left(k a^{\prime}\right)-b_{n}^{B} H_{n}^{(2)}\left(k a^{\prime}\right)\right|^{2}}{\sum_{n=-\infty}^{\infty}\left|a_{n} H_{n}^{(1)}\left(k a^{\prime}\right)\right|^{2}}
$$

In practice we truncate the series using $-10 \leq n \leq 10$. Arbitrarily we choose $k=1$ and $a^{\prime}=.1$. We plot the error as a function of $\tilde{\rho}$ in Figure 1 . When $\tilde{\rho}$ is real (i.e. when $\sigma=0$ ) there are eigenvalues for the annular domain and

$\mathrm{RR} \mathrm{n}^{\circ} 3049$ 
these show up clearly as peaks in the error surface. As the imaginary part of $\tilde{\rho}$ increases the error decreases and can be made arbitrarily small if the imaginary part is large enough. In Figure 2 we show the error as a function of the real part of $\tilde{\rho}$ for fixed imaginary part. By deciding on a desired error level, one can find an appropriate value of $\tilde{\rho}_{b}$.

In the subsequent sections we actually use the Robin boundary condition on the outer boundary. In this case (for the circular scatterer of radius $a^{\prime}$ ) we solve the following problem:

$$
\begin{aligned}
\nabla \cdot\left(A \nabla \hat{H}^{B}\right)+k^{2} d \bar{d} \hat{H}^{B} & =0 \text { for } a^{\prime}<\rho<b \\
\frac{\partial \hat{H}^{B}}{\partial \rho} & =\frac{\partial \exp (i k x)}{\partial \rho} \text { when } \rho=a^{\prime} \\
\frac{\partial \hat{H}^{B}}{\partial \rho}-i k d \hat{H}^{B} & =0 \text { when } \rho=b
\end{aligned}
$$

This problem has the solution

$$
\hat{H}^{B}(\rho, \theta)=\sum_{n=-\infty}^{\infty}\left(a_{n}^{B} H_{n}^{(1)}(k \tilde{\rho})+b_{n}^{B} H_{n}^{(2)}(k \tilde{\rho})\right) \exp (i n \theta)
$$

where

$$
a_{n}^{B}=-\frac{H_{n}^{(2) \prime}\left(k \tilde{\rho}_{b}\right)-i H_{n}^{(2)}\left(k \tilde{\rho}_{b}\right)}{H_{n}^{(1) \prime}\left(k \tilde{\rho}_{b}\right)-i H_{n}^{(1)}\left(k \tilde{\rho}_{b}\right)} b_{n}^{B} \text { and } b_{n}^{B}=-\frac{i^{n} J_{n}^{\prime}\left(k a^{\prime}\right)}{W}
$$

provided $W \neq 0$ where

$$
W=H_{n}^{(2) \prime}\left(k a^{\prime}\right)-\frac{H_{n}^{(2) \prime}\left(k \tilde{\rho}_{b}\right)-i H_{n}^{(2)}\left(k \tilde{\rho}_{b}\right)}{H_{n}^{(1) \prime}\left(k \tilde{\rho}_{b}\right)-i H_{n}^{(1)}\left(k \tilde{\rho}_{b}\right)} H_{n}^{(1) \prime}\left(k a^{\prime}\right) .
$$

When $W=0$ the problem is not uniquely solvable. We could not prove existence or uniqueness for this Bérenger problem in general but our calculations suggest that for the annulus existence and uniqueness only fail at $k=0$ (where the problem becomes a pure Neumann problem). This accounts for the error spike towards $\tilde{\rho}=0$ shown in Figure 3. Compared to Figure 1 we can see that there are no other eigenvalues for the range of $\tilde{\rho}$ considered here. The error curves shown in Figure 4 (compared to Figure 2) show that the error obtained using the Robin boundary condition is much smaller than the error obtained using Dirichlet boundary conditions. This justifies our use of the Robin condition in the numerical results presented later in this section. 


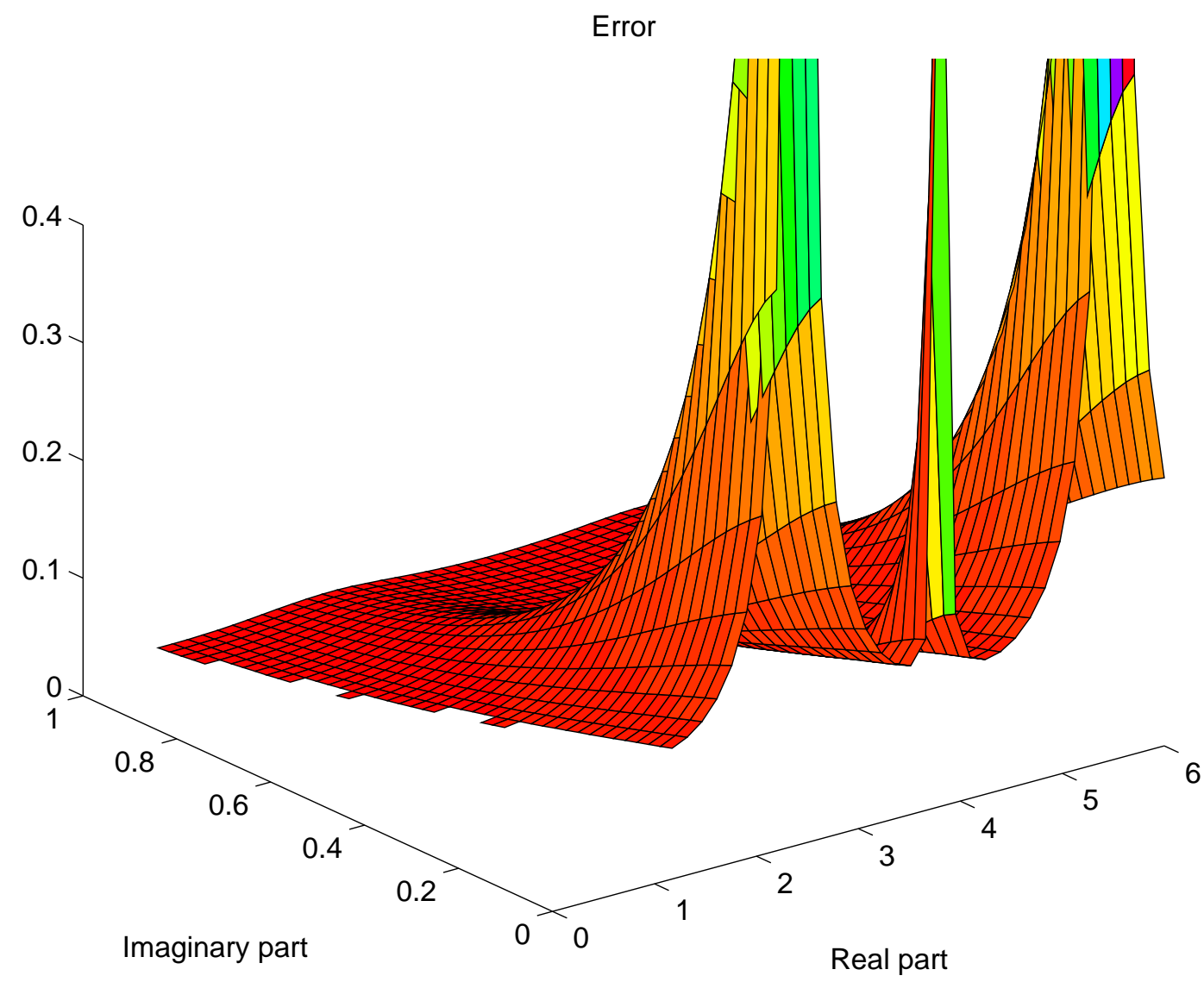

Figure 1: Here we plot the relative $L_{2}$ error defined by (46) between the exact solution of the time harmonic scattering problem for a circle and the Bérenger solution using a Dirichlet outer boundary. We show the error as a function of $\tilde{\rho}_{b}$. When $\tilde{\rho}_{b}$ is real there are eigenvalues for the annular domain and these show up as peaks in the error surface. As the imaginary part of $\tilde{\rho}_{b}$ increases, the error decreases. Away from the real axis we see no evidence of lack of existence or uniqueness for the Bérenger problem. 

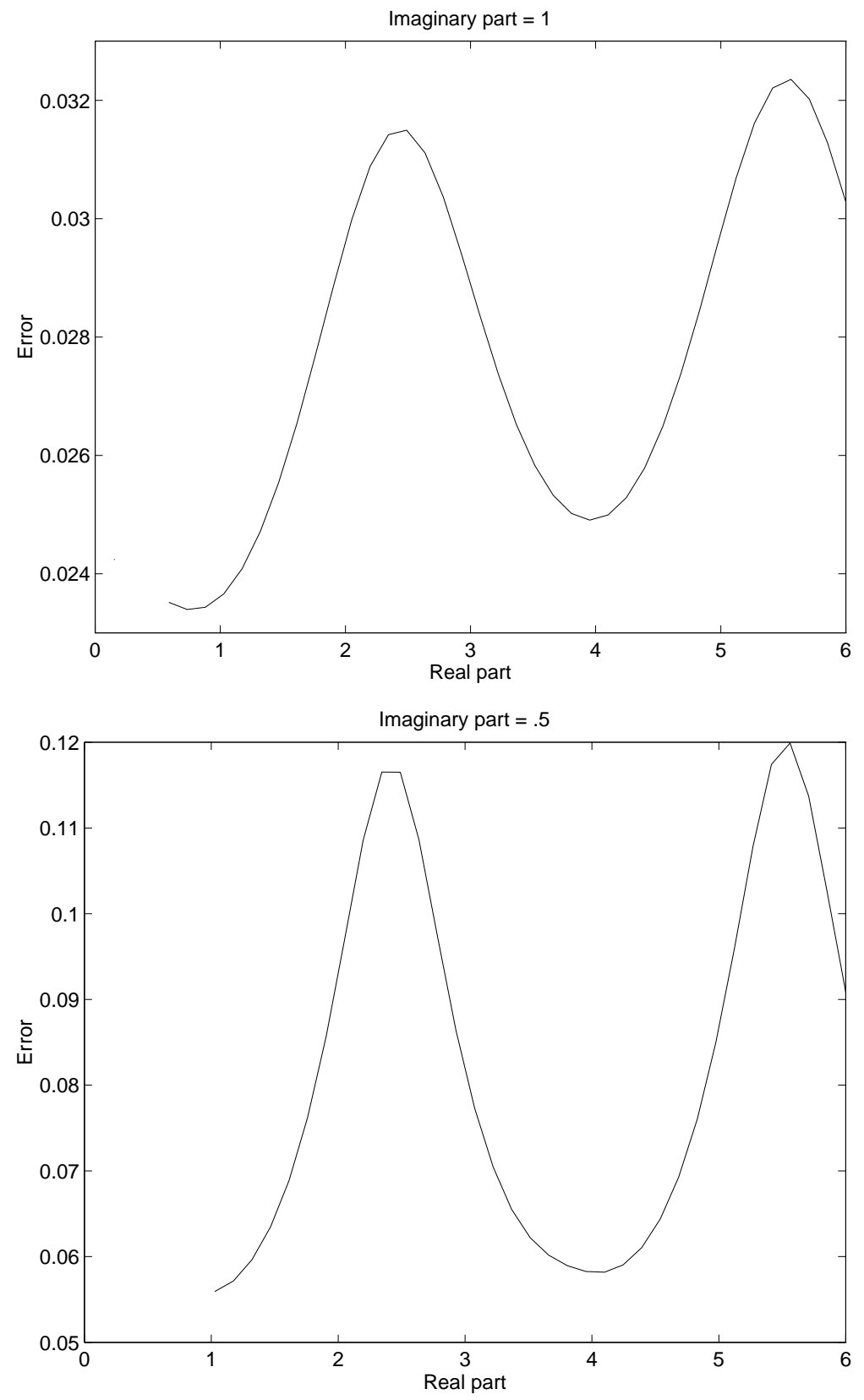

Figure 2: Here we plot the relative $L_{2}$ error defined by (46) between the exact solution of the time harmonic scattering problem for a circle and the Bérenger solution using a Dirichlet outer boundary. We show the error as a function of $\Re\left(\tilde{\rho}_{b}\right)$. In the top panel $\Im\left(\tilde{\rho}_{b}\right)=1$ and in the bottom panel $\Im\left(\tilde{\rho}_{b}\right)=.5$. When $\Im\left(\tilde{\rho}_{b}\right)=1$ the error is reduced to approximately $3 \%$. 


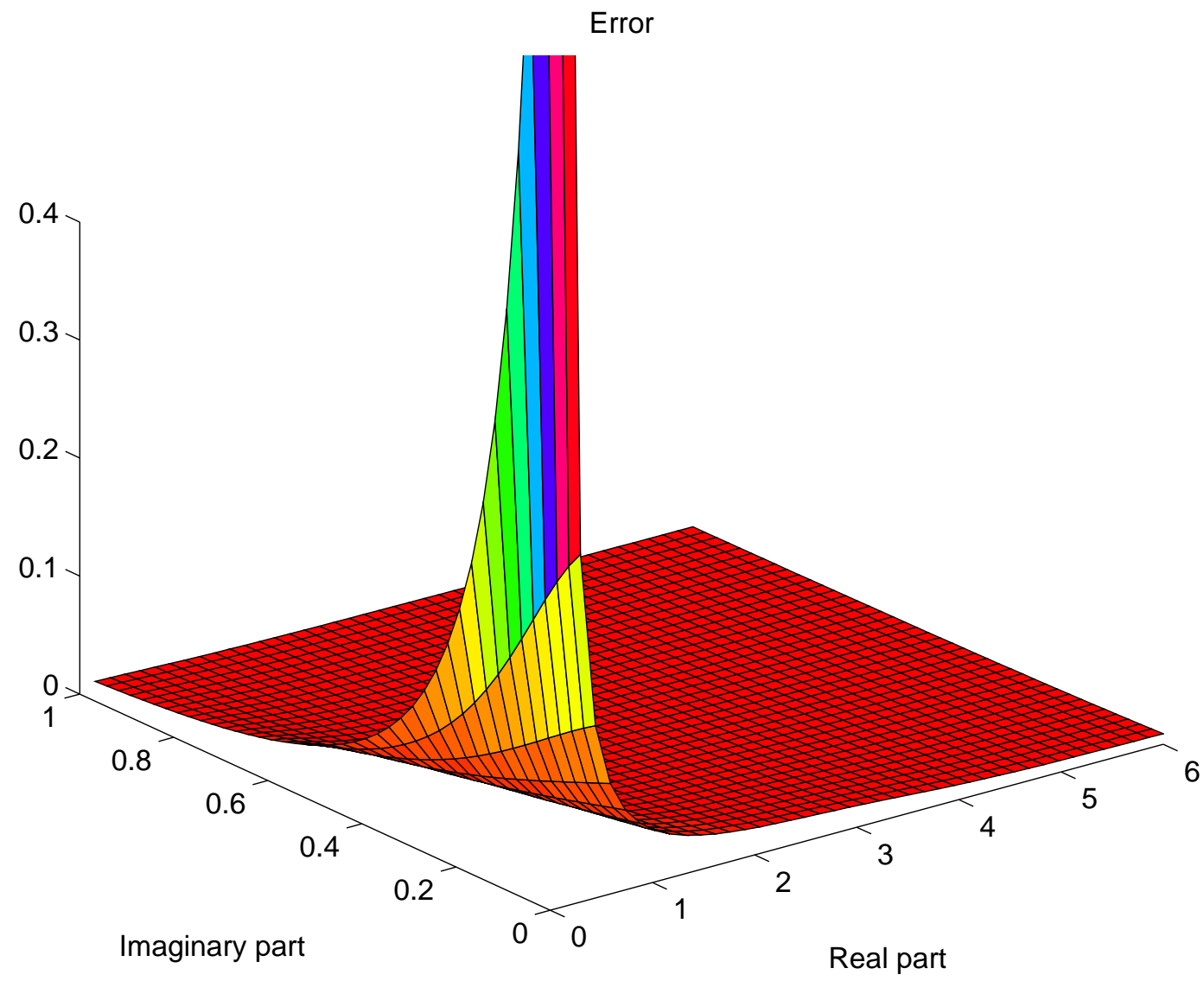

Figure 3: Here we plot the relative $L_{2}$ error defined by (46) between the exact solution of the time harmonic scattering problem for a circle and the Bérenger solution using a Robin outer boundary. We show the error as a function of $\tilde{\rho}_{b}$. For $\tilde{\rho}_{b}$ small the error is large but for a sufficiently large real or imaginary part the error can be made arbitrarily small. Apart from when $k=0$, we see no evidence of lack of existence or uniqueness for the Bérenger problem. 

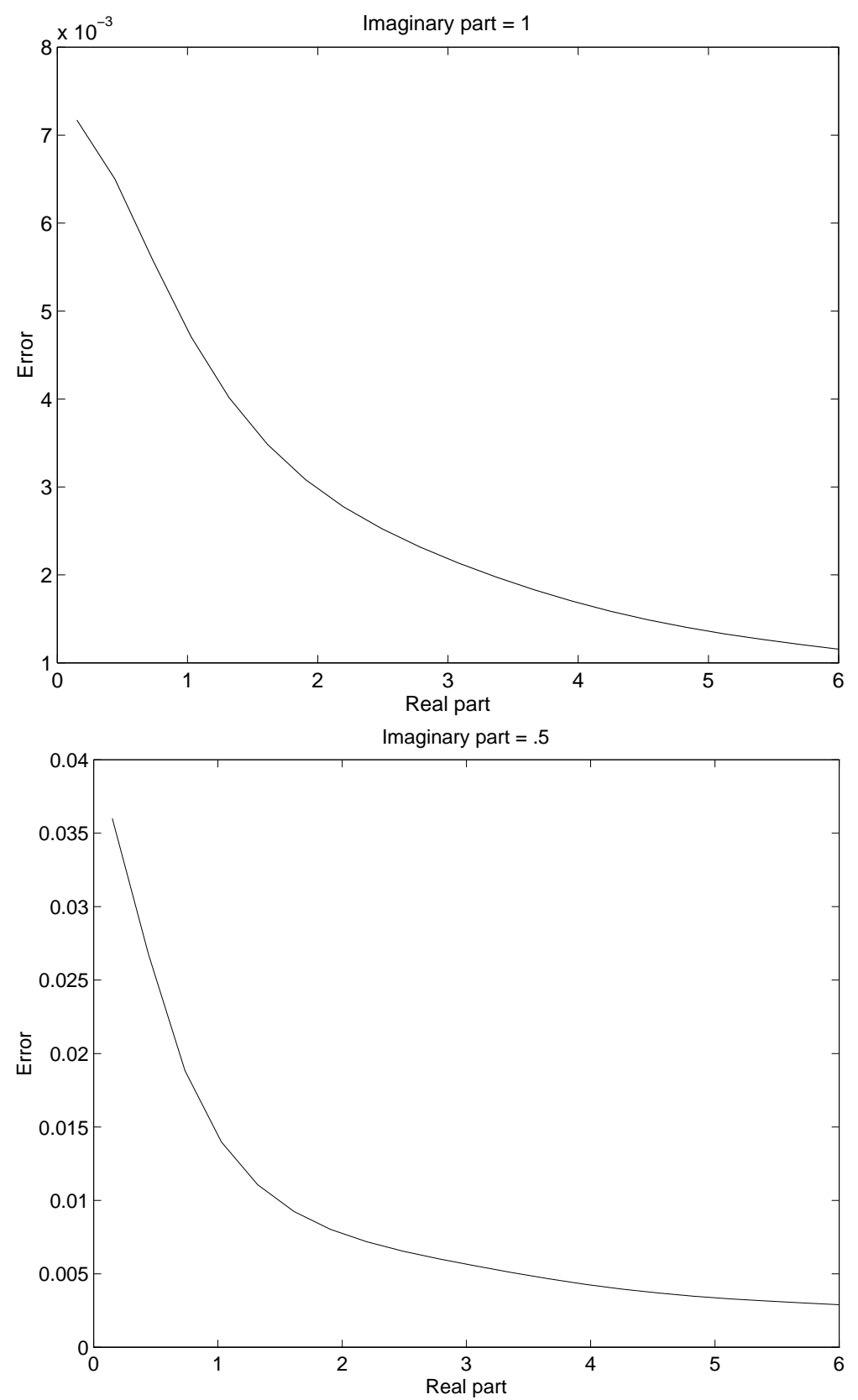

Figure 4: Here we plot the relative $L_{2}$ error defined by (46) between the exact solution of the time harmonic scattering problem for a circle and the Bérenger solution using a Robin outer boundary. We show the error as a function of $\Re\left(\tilde{\rho}_{b}\right)$. In the top panel $\Im\left(\tilde{\rho}_{b}\right)=1$ and in the bottom panel $\Im\left(\tilde{\rho}_{b}\right)=.5$. Compared to Figure 2 we see that the use of a Robin condition on the outer boundary improves the error in the Bérenger solution. For example we can reduce the error to approximately $3 \%$ by using $\Im\left(\tilde{\rho}_{b}\right)=.5$ instead of $\Im\left(\tilde{\rho}_{b}\right)=1$ when we use the Dirichlet boundary condition. 


\subsection{Time harmonic problems}

The purpose of this section is to show that the Cartesian and cylindrical Bérenger PML layers can be used to compute near field solutions of the Helmholtz equation (and hence by a suitable transformation the far field). We shall only show the results of one numerical experiment since we will provide a more detailed numerical test of the curvilinear method for the time domain problem (where the Bérenger method is most popular and where the behavior of the Cartesian layer is well known).

For this test, we compute the field scattered from a perfectly conducting metal obstacle in transverse polarization. This corresponds to Neumann data imposed on the metal wall. The incoming wave is $\hat{H}^{M(i)}=\exp (i k x)$. The scatterer is contained in the box $-2<x<2.2$ and $-0.7<y<0.7$ and the speed of light to be unity so wave number is $k=\omega=6.2832$. The grid used is shown in Figure 5 where the outer radius of the circle is $\rho=5$ and the maximum diameter of elements in the mesh is $h=0.279$. Note that the grid is not aligned with Cartesian or radial coordinate system, which is convenient from the point of view of using a standard mesh generator. Cubic isoparametric elements are used to discretize the problem. For the Cartesian case we use a simple Newumann boundary condition on the outer boundary (it would be more usual in this case to use a rectangular outer boundary but we want to make comparisons with a single grid). For the curvilinear PML we use the modified Sommerfeld radiation condition being used on the outer boundary (ie., we are solving the Robin problem (26), (27) and (31)). We choose $\sigma(s, a)=\sigma_{0}(|s|-a)^{2}$ for $|s|>a$ and $\sigma=0$ for $|s| \leq a$. In all cases we choose $\sigma_{0}=5$.

In the Cartesian case we use

$$
d_{x}(x)=1+\frac{i}{\omega} \sigma(x, 2.5) \text { and } d_{y}(y)=1+\frac{i}{\omega} \sigma(y, 1.2) .
$$

This implies that the layer is half a distance unit from the scatterer. For the curvilinear case we use

$$
d(r)=1+\frac{i}{\omega} \sigma(r, 2.5)
$$

Figure 6 shows contours of the real part of $\hat{H}^{M}$ computed by a capacitance matrix technique that matches the finite element solution to an integral equation solution outside the grid (thus handling the infinite domain accurately)

$\mathrm{RR} \mathrm{n}^{\circ} 3049$ 


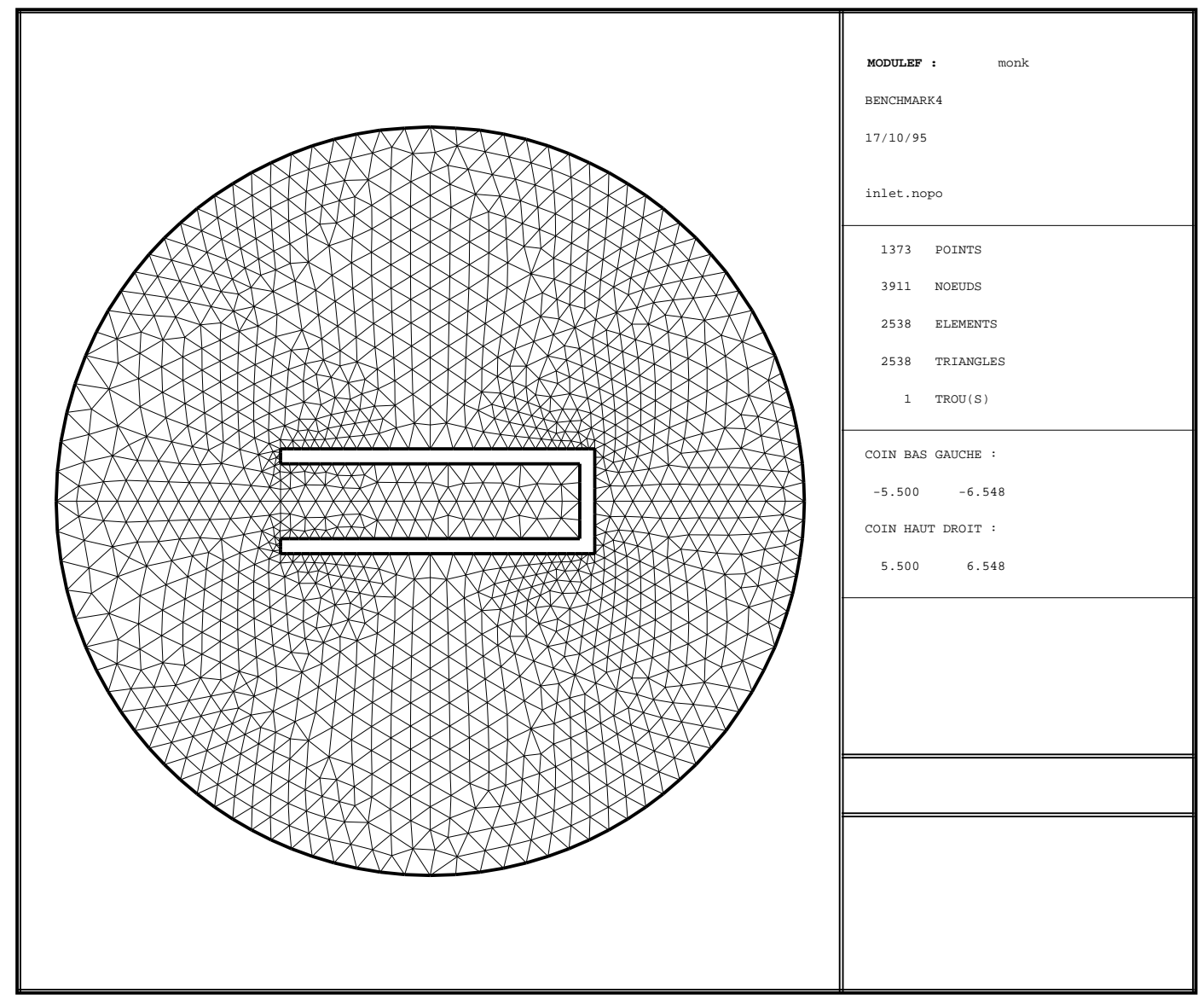

Figure 5: The mesh used in the numerical experiments reported on the time harmonic wave equation. 


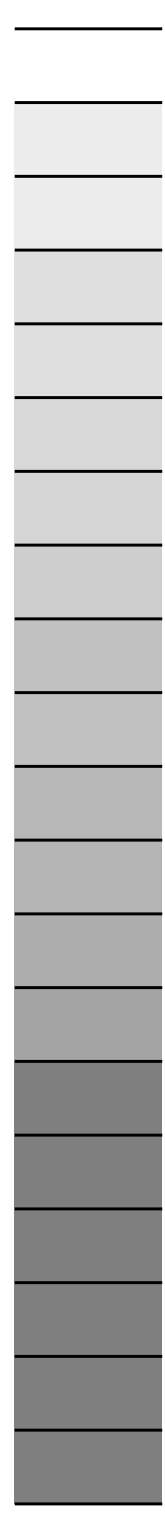

1.429719

1.286171

1.142623

0.999075

0.855527

0.71198

0.568432

0.424884

0.281336

0.137788

$-0.00576$

$-0.149308$

$-0.292856$

$-0.436404$

$-0.579952$

$-0.723499$

$-0.867047$

$-1.010595$

$-1.154143$

$-1.297691$

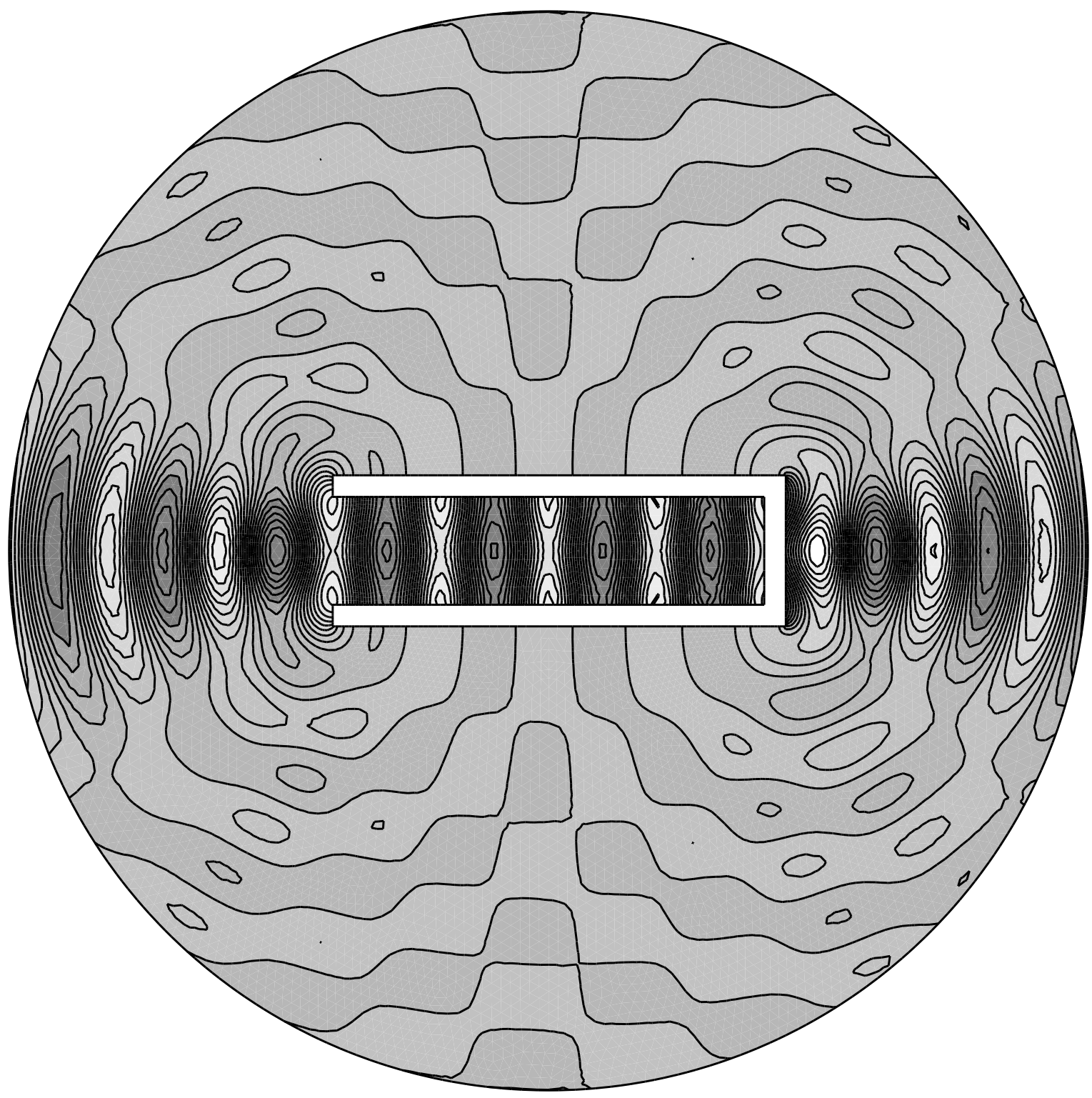

Figure 6: The real part of the scattered field computed using a matched finite element - integral equation code. 


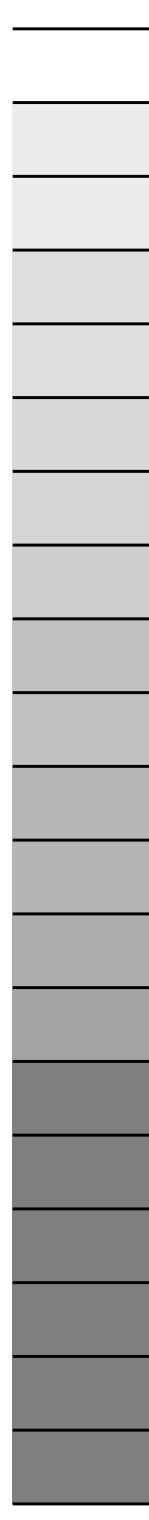

1.429247

1.285883

1.142518

0.999154

0.85579

0.712425

0.569061

0.425697

0.282332

0.138968

$-0.004396$

$-0.147761$

$-0.291125$

$-0.434489$

$-0.577853$

$-0.721218$

$-0.864582$

$-1.007946$

$-1.151311$

$-1.294675$

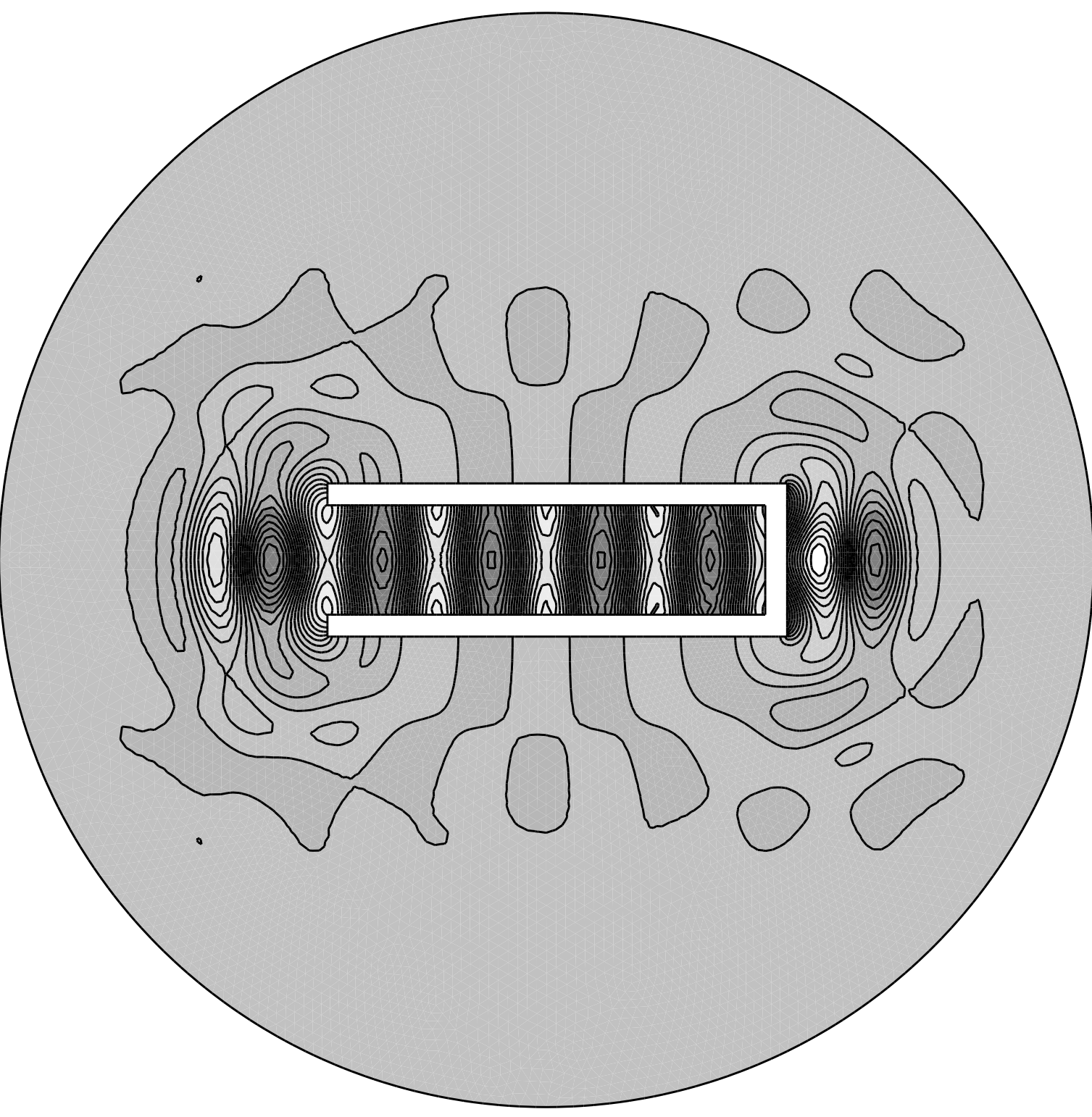

Figure 7: The real part of the scattered field computed using the Cartesian Bérenger absorbing layer. 


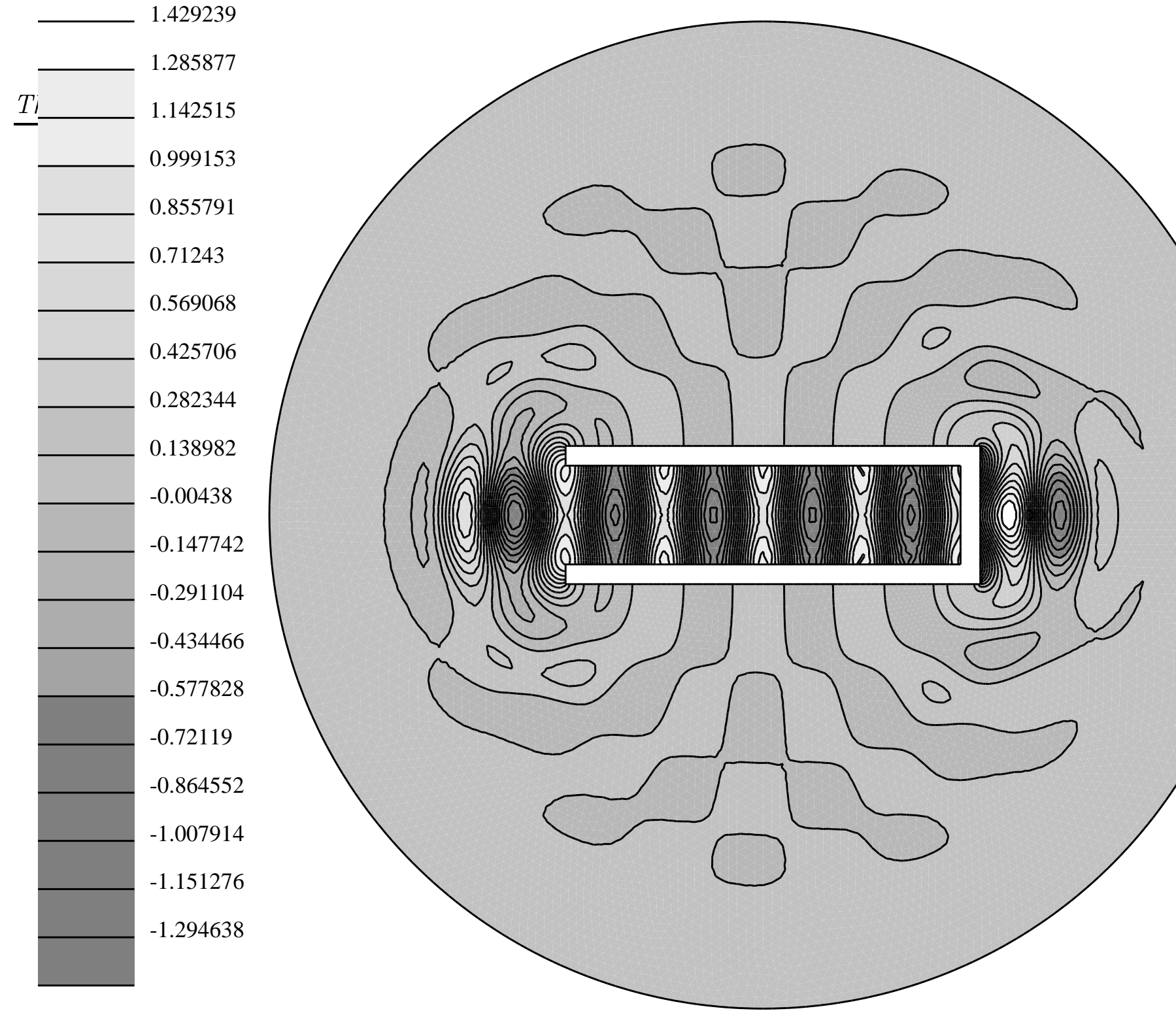

Figure 8: The real part of the scattered field computed using the curvilinear Bérenger absorbing layer. 
[19]. Figure 7 shows the real part of the Bérenger solution $\hat{H}^{B}$ using the Cartesian layer, and 8 shows the real part of the Bérenger solution $\hat{H}^{B}$ using the curvilinear layer. Clearly the near field (for example in the scatterer) computed using both Bérenger layers is similar to the capacitance matrix solution in Figure 6. But, as expected, the Bérenger solution dies away rapidly in the absorbing layer. Furthermore, in both cases, the contours of the solution show no abrupt curvature changes when the Bérenger layer is entered which indicate that the layer is "perfectly" matched.

Figure 9 shows the real part of the scattered field along the $x$ axis computed using the matched finite element and integral equation approach. Figure 10 and 11 shows the corresponding result for the Cartesian and curvilinear Bérenger media. The solutions are similar for $|x|<2.5$ (this is for coordinate values from 2.5 to 7.5 on the one dimensional plots) but the Bérenger result dies away quickly once the Bérenger medium is reached. These results give numerical support to the claim that the rectilinear and curvilinear Bérenger method can be used to compute time harmonic solutions.

\subsection{Time Dependent System}

In this section we shall investigate in some detail the curvilinear Bérenger method for the time dependent problem. Figure 12 shows a schematic of the grid used for the numerical tests of the curvilinear PML. Inside the circle of radius $\rho=a$ we use isoparametric quadrilateral Nédélec elements without lumping [22]. Outside for $a<\rho<b$, we use isoparametric quadrilateral Nédélec elements with an extra unknown associated with the centroid of the elements. The curved segments of the mesh (for example in the Bérenger layer) are approximated by piecewise linear approximations. This means that there is some geometric error in approximating the Bérenger layers.

The arrangement of degrees of freedom for the elements is shown in Figure 13. The magnetic degrees of freedom (for $H$ or $H^{(\rho)}$ ) are associated with the centroid of the elements and we use mapped piecewise constant basis functions for the fields. The electric degrees of freedom for $E_{\rho}$ and $E_{\theta}$ are associated with edges of the mesh. 


\section{Matched FE/Nystrom}

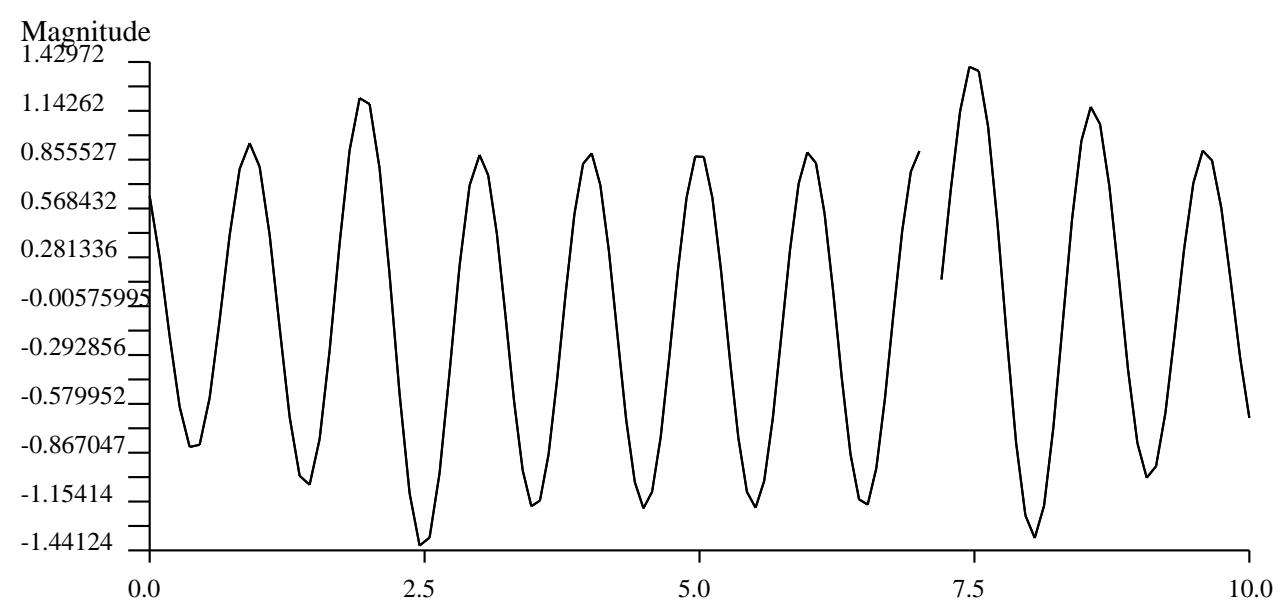

Figure 9: The real part of the scattered field computed using the matched finite element - integral equation approach. Here we plot the solution along the line $y=0$ (zero on the $x$ axis corresponds to the left outer boundary of the domain). The break in the solution is due to the perfectly conducting scatterer.

$\mathrm{RR} \mathrm{n}^{\circ} 3049$ 


\section{Berenger, Cartesian PML}

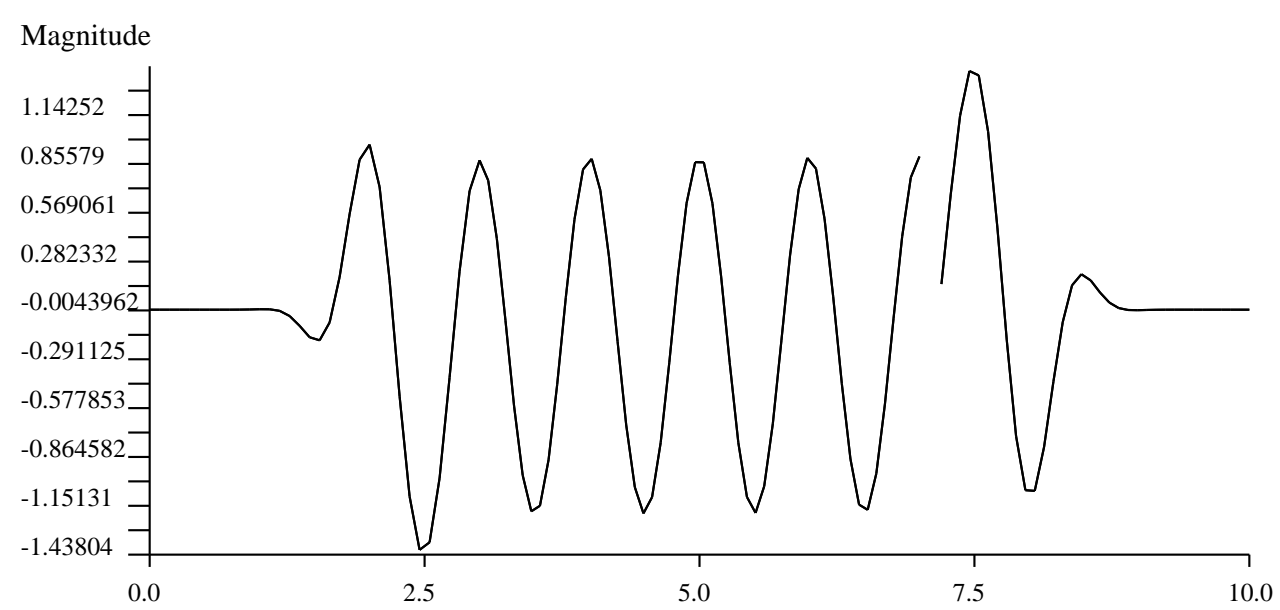

Figure 10: The real part of the scattered field computed using the Cartesian Bérenger absorbing medium. Here we plot the solution along the line $y=0$. The break in the solution is due to the perfectly conducting scatterer. 


\section{Berenger, Curvilinear PML}

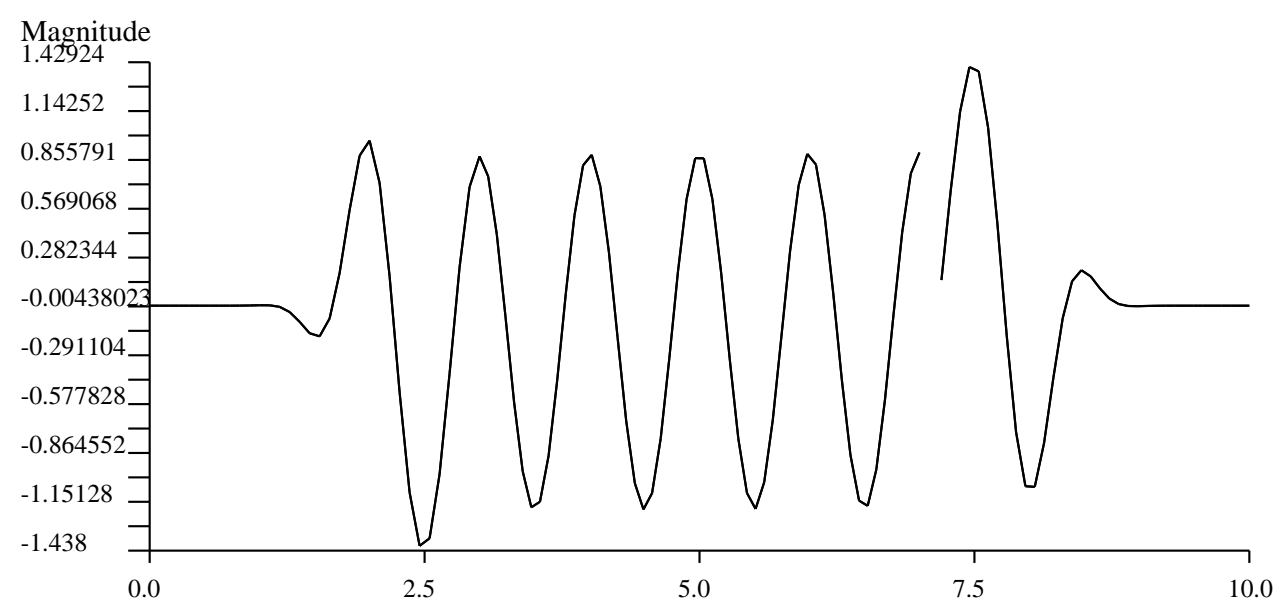

Figure 11: The real part of the scattered field computed using the curvilinear Bérenger absorbing medium. Here we plot the solution along the line $y=0$. The break in the solution is due to the perfectly conducting scatterer.

$\mathrm{RR} \mathrm{n}^{\circ} 3049$ 


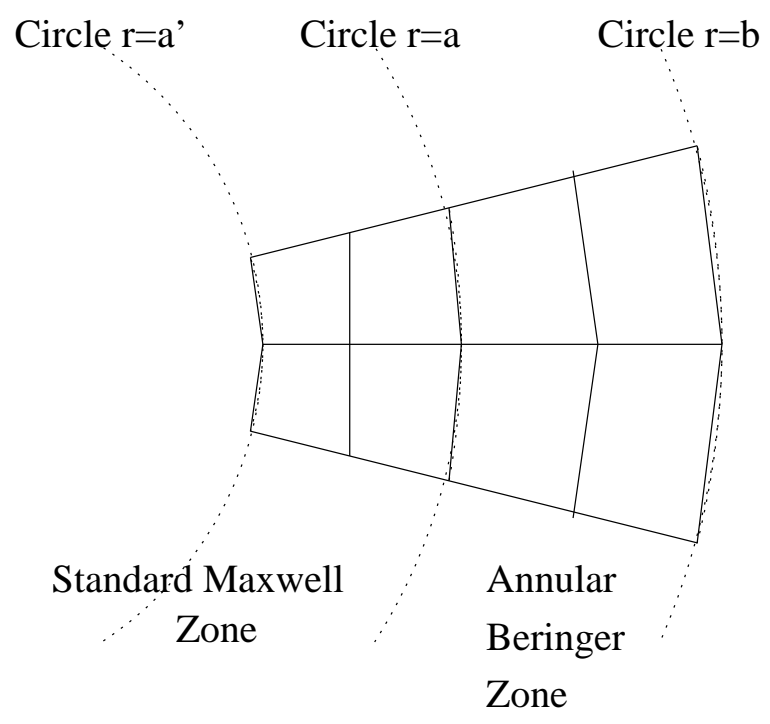

Figure 12: A schematic of the grid used. Inside the circle $\rho=a$ up to the scatterer we use standard linear Nédélec elements on isoparametric quadrilaterals. Outside $r=a$, four sided isoparametric Bérenger elements are used. 


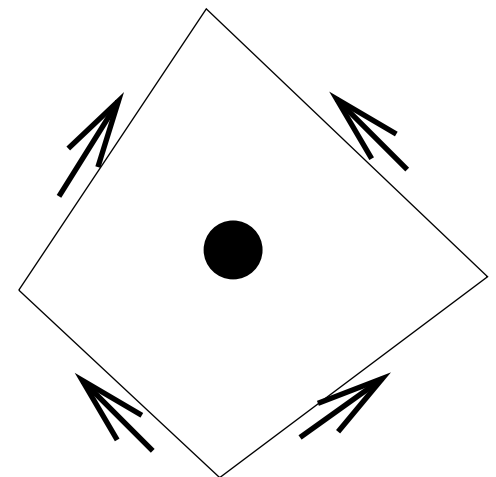

(a)

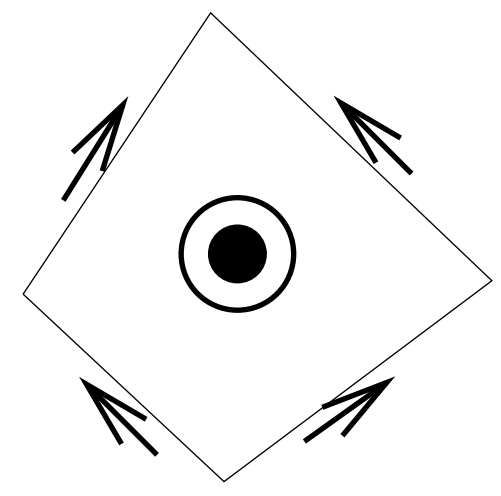

(b)

Figure 13: A schematic showing the arrangement of degrees of freedom for the elements. The electric field unknowns are associated with edges in the mesh. The magnetic field unknowns are associated with centroids of the elements. (a) Standard Nédélec element, (b) Bérenger element.

On the outer edge of the domain (when $|\boldsymbol{x}|=b$ ) we use the first order absorbing boundary condition:

$$
H+\chi \boldsymbol{n} \times \boldsymbol{E}=0 \text { on }|\boldsymbol{x}|=b,
$$

where $\chi=\sqrt{\epsilon \mu}$. Note that this boundary condition is note the same as the Sommerfeld absorbing boundary condition used in the frequency domain calculations, but it is easier to implement for finite element methods.

Now let us detail the discrete problem. Let $D_{b}$ denote the bounded domain including the Bérenger layer. Let $\tau_{h}$ be a mesh of linear isoparametric quadrilaterals of maximum edge length $h$ approximating the domain $D_{b}$ as described above. Then the finite element space $U_{h}$ satisfies $U_{h} \subset H(\operatorname{curl} ; \Omega)$ and is constructed using the standard space of isoparametric linear edge elements. Let $U_{h, 0}=\left\{\boldsymbol{u} \in U_{h} \mid \boldsymbol{n} \times \boldsymbol{u}=0\right.$ on $\left.\Gamma\right\}$ where $\Gamma$ is the surface of the scatterer (in the results shown later the boundary is the circle of radius 0.1). Let $V_{h}$ be the corresponding set of mapped piecewise constant functions containing $\nabla \times U_{h}$. Let $\Delta t>0$ represent the time step (which must be sufficiently small compared to $h$ for stability). Then we seek $\boldsymbol{E}_{h}^{n} \in U_{h}, H_{h}^{n+1 / 2} \in V_{h}$ and 
$H_{h}^{(\rho), n+1 / 2} \in V_{h}$ for $n=0,1, \cdots$ such that $\boldsymbol{E}_{h}^{0}$ and $H_{h}^{1 / 2}$ approximate the fields at $t=0$ and $t=\Delta t / 2$ respectively (the field $H_{h}^{1 / 2}$ is computed from $\boldsymbol{E}_{h}^{0}$ and $H_{h}^{0}$ by a Runge-Kutta step applied to the Maxwell system) and $H_{h}^{(\rho), 1 / 2}=0$. Then $\boldsymbol{E}_{h}^{n+1} \in U_{h}$ satisfies (we denote by $(.,$.$) the L_{2}(\Omega)$ inner product for vector or scalar functions and use standard $x$ and $y$ components of the electric field):

$$
\begin{aligned}
& \left(\epsilon\left(\frac{\boldsymbol{E}_{h}^{n+1}-\boldsymbol{E}_{h}^{n}}{\Delta t}\right), \boldsymbol{\psi}_{h}\right)+\left(\epsilon \boldsymbol{\sigma}\left(\frac{\boldsymbol{E}_{h}^{n+1}+\boldsymbol{E}_{h}^{n}}{2}\right), \boldsymbol{\psi}_{h}\right)+ \\
& \left\langle\chi \boldsymbol{n} \times\left(\frac{\boldsymbol{E}_{h}^{n+1}+\boldsymbol{E}_{h}^{n}}{2}\right), \boldsymbol{n} \times \boldsymbol{\psi}_{h}\right\rangle_{b} \\
& \quad=\left(\nabla \times H_{h}^{n+1 / 2}, \boldsymbol{\psi}_{h}\right)-\left(\boldsymbol{J}^{n+1 / 2}, \boldsymbol{\psi}_{h}\right) \quad \forall \boldsymbol{\psi}_{h} \in U_{h, 0} \\
& \boldsymbol{n} \times \boldsymbol{E}_{h}^{n+1}=\boldsymbol{\gamma}_{h}^{n+1 / 2} \text { on } \Gamma
\end{aligned}
$$

where (if $x=r \cos (\theta)$ and $y=r \sin (\theta)$ )

$$
\boldsymbol{\sigma}=\left(\begin{array}{cc}
\sigma \sin ^{2}(\theta)+\bar{\sigma} \cos ^{2}(\theta) & (\bar{\sigma}-\sigma) \cos (\theta) \sin (\theta) \\
(\bar{\sigma}-\sigma) \cos (\theta) \sin (\theta) & \sigma \cos ^{2}(\theta)+\bar{\sigma} \sin ^{2}(\theta)
\end{array}\right) .
$$

Next $H_{h}^{(\rho), n+1 / 2} \in V_{h}$ is computed via

$$
\begin{aligned}
& \left(\mu\left(\frac{H_{h}^{(\rho), n+3 / 2}-H_{h}^{(\rho), n+1 / 2}}{\Delta t}\right), \phi_{h}\right)+\left(\mu \sigma\left(\frac{H_{h}^{(\rho), n+3 / 2}+H_{h}^{(\rho), n+1 / 2}}{2}\right), \phi_{h}\right) \\
= & -\left(\frac{\partial}{\partial \rho} E_{\theta, h}^{n+1}, \phi_{h}\right) \quad \forall \phi_{h} \in V_{h},
\end{aligned}
$$

where $E_{\theta, h}^{n+1}$ denotes the $\theta$ component of $\boldsymbol{E}_{h}^{n+1}$. This computation is only carried out in the Bérenger layer (the derivative of $E_{\theta, h}^{n+1}$ is well defined by virtue of having an annular grid in the Bérenger layer).

Finally $H_{h}^{n+3 / 2} \in V_{h}$ is given by

$$
\begin{gathered}
\left(\mu\left(\frac{H_{h}^{n+3 / 2}-H_{h}^{n+1 / 2}}{\Delta t}\right), \phi_{h}\right)+\left(\mu \bar{\sigma}\left(\frac{H_{h}^{n+3 / 2}+H_{h}^{n+1 / 2}}{2}\right), \phi_{h}\right) \\
+\left(\mu(\sigma-\bar{\sigma}) H_{h}^{(\rho), n+3 / 2}, \phi_{h}\right)=-\left(\nabla \times \boldsymbol{E}_{h}^{n+1}, \phi_{h}\right) \quad \forall \phi_{h} \in V_{h} .
\end{gathered}
$$


Note that we have not used a centered difference for the Bérenger term here. Numerical experiments show that this choice gives a better solution than the standard symmetric average of Bérenger variables, but is likely to be method dependent.

\subsection{Scattering from a circle.}

We consider an infinite domain problem of scattering of a plane wave off a circular perfect conductor. We assume that the incident plane wave is given by

$$
\begin{aligned}
& \boldsymbol{E}_{i}(\boldsymbol{x}, t)=\left(\begin{array}{c}
0 \\
\sqrt{\mu_{0} / \epsilon_{0}} g\left(\left(t-(x-0.1) \sqrt{\epsilon_{0} \mu_{0}}\right) 10^{9}\right)
\end{array}\right) \\
& H_{i}(\boldsymbol{x}, t)=g\left(\left(t-(x-0.1) \sqrt{\epsilon_{0} \mu_{0}}\right) 10^{9}\right) \text {. }
\end{aligned}
$$

where $g$ is given by

$$
g(s)= \begin{cases}\frac{\exp \left(-10(s-1)^{2}\right)-\exp (-10)}{1-\exp (-10)} & 0 \leq s \leq 2 \\ 0 & s>2 \text { or } s<0 .\end{cases}
$$

This wave is incident on a perfectly conducting circular cylinder of radius $0.1 \mathrm{~m}$ centered at the origin. We choose

$$
\begin{aligned}
\epsilon=\epsilon_{0} & =8.85 \times 10^{-12} \mathrm{~F} / \mathrm{m}^{2} \\
\mu=\mu_{0} & =1.2566 \times 10^{-6} \mathrm{~N} / \mathrm{A}^{2} .
\end{aligned}
$$

Using special function theory, an exact solution is available for this problem $[17]$.

For the numerical problem we take an annular domain with inner radius $0.1 \mathrm{~m}$ and an outer radius of $0.3 \mathrm{~m}$. On $r=0.1$ we impose $\boldsymbol{n} \times \boldsymbol{E}=\gamma \equiv-\boldsymbol{n} \times \boldsymbol{E}_{i}$, which describes how the incident field scatters off of a perfect conductor. The grid used has mesh points distributed uniformly in the $(r, \theta)$ plane, i.e. the mesh points are $\left(r_{i}, \theta_{j}\right), 0 \leq i \leq N_{r}, 0 \leq j<N_{\theta}$, where

$$
r_{i}=0.1+0.2 \frac{i}{N_{r}} \quad \text { and } \theta_{j}=2 \pi \frac{j}{N_{\theta}} .
$$

$\mathrm{RR} \mathrm{n}^{\circ} 3049$ 


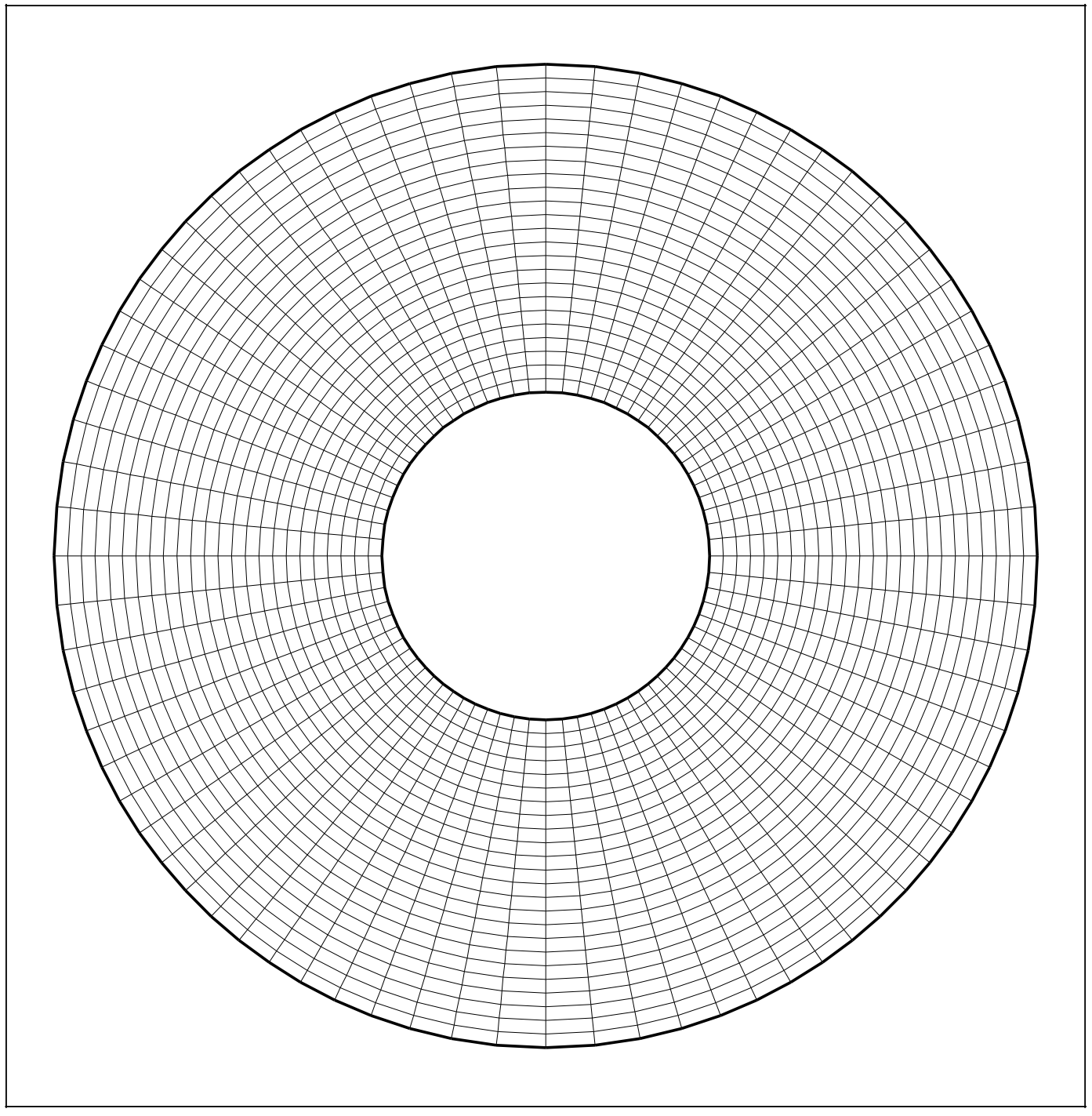

Figure 14: The base mesh used in the numerical experiments reported later on the time dependent Maxwell system. Additional layers of cells are added to form the Bérenger layer. 
A typical grid is shown in Figure 14 with $N_{r}=25$ and $N_{\theta}=80$. This is the base mesh used for the calculations shown next.

The Bérenger boundary layer is implemented by adding extra layers of cells outside the base mesh. We use a total of $N_{\text {layer }}$ layers. The radial size the these cells is the $0.2 / N_{r}$ (ie. the same as for the cells in the base mesh). The time step used is $\Delta t=0.01$ and the mesh size (measured as the longest edge in a quadrilateral) ranges from approximately $h=0.007$ near the scatterer to $h=0.03$ on the outer layer of the base mesh. The solution is computed for $0 \leq t \leq 7$.

In Figure 15 we show a plot of $H(x, y, t)$ against $t$ at the point $(x, y)=$ $(-0.206,-0.0104)$. This is a grid point for the magnetic field. We can compute a solution that is not degraded by mesh termination by computing on a grid in which 150 extra layers have been added to the base mesh and in which the Bérenger variables are set to zero. In this case the relative $L_{2}$ error is $0.18 \%$. Using the simple first order ABC (with 9 extra layers) gives a relative error of $15.5 \%$. We have tried three layers shown in Table 1. In each case $\sigma$ is computed by

$$
\sigma_{i}=\sigma_{0} \gamma^{i-1} / h_{l}
$$

where $\sigma_{0}$ is the value in the first Bérenger layer, $i$ is the index of the layer, $\gamma$ is the growth factor, and $h_{l}$ is the radial mesh size in the layer.

From Table 2 we can see that our time domain results produce solutions that have less than a tenth of the error of the first order absorbing boundary condition results. Unfortunately, with the time step of $\Delta t=0.01$ the error in the Bérenger solution is about four times the error in the underlying finite element method. With a smaller time step this can be reduced to approximately twice the underlying error suggesting that the phase error in the finite element method is polluting the results.

\section{Conclusion.}

In this paper we have presented a method for implementing the Bérenger PML in curvilinear coordinates. Our theory and numerical experiments suggest that such a layer can be used in the frequency domain with a standard finite element method. In the time domain the layer can also be used and gives more accurate

$\mathrm{RR} \mathrm{n}^{\circ} 3049$ 


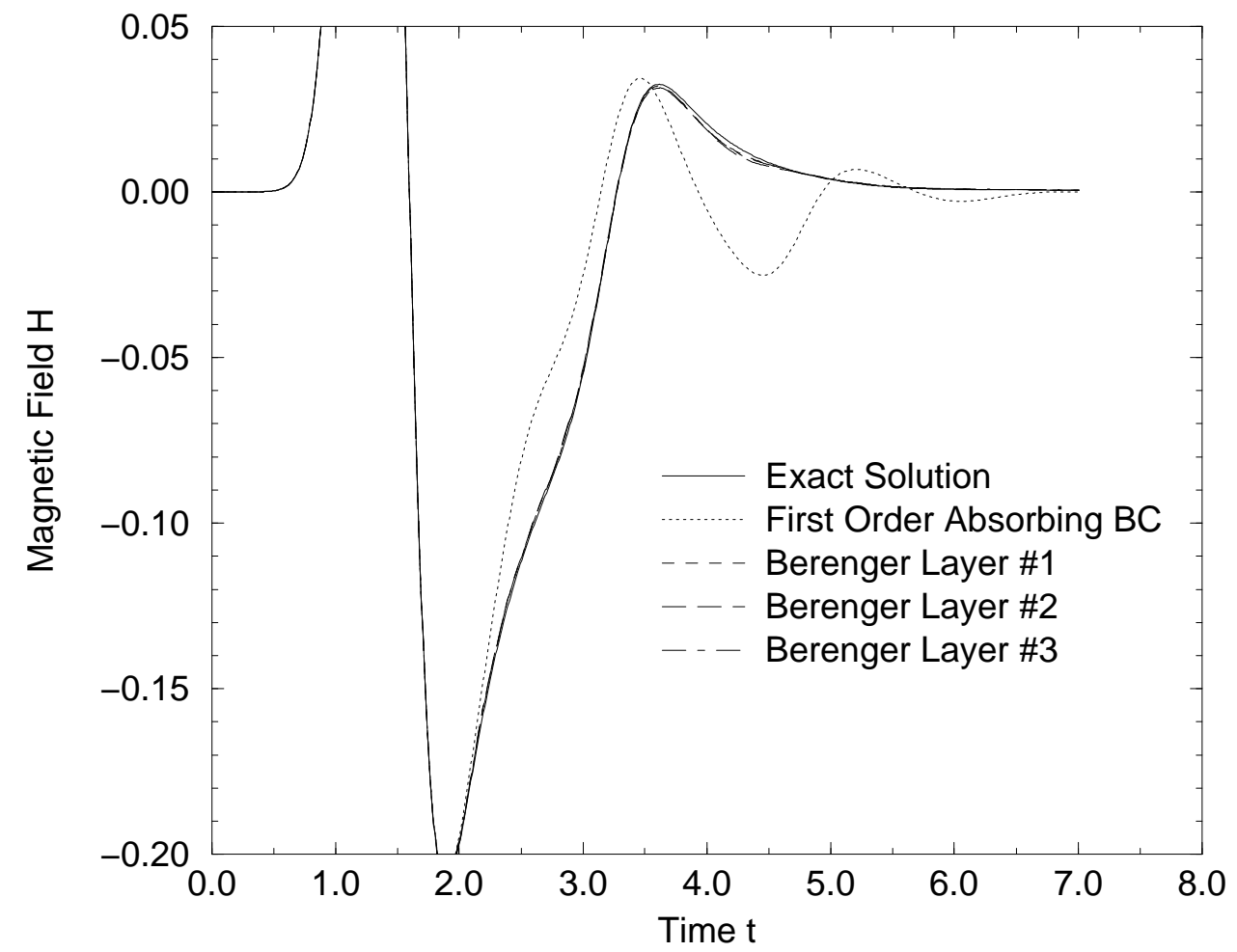

Figure 15: A graph of $H(x, y, t)$ against $t$ at $(x, y)=(-0.206,-0.0104)$. We show the result of using a three different Bérenger layers with 9 shells in each layer. The results are compared to an exact solution and the solution computed using a first order absorbing boundary condition. 


\begin{tabular}{||r|lll||}
\hline \hline Index of shell & layer 1 $(\gamma=1.6)$ & layer $2(\gamma=1.6)$ & layer $3(\gamma=1.6)$ \\
\hline 1 & 0.02 & 0.04 & 0.02 \\
2 & 0.032 & 0.064 & 0.032 \\
3 & 0.0512 & 0.1024 & 0.0512 \\
4 & 0.08192 & 0.16384 & 0.08192 \\
5 & 0.1310720 & 0.262144 & 0.1310720 \\
6 & 0.2097152 & 0.4194304 & 0.2097152 \\
7 & 0.2097152 & 0.4194304 & 0.3355443 \\
8 & 0.2097152 & 0.4194304 & 0.5368709 \\
9 & 0.2097152 & 0.4194304 & 0.8589935 \\
\hline \hline
\end{tabular}

Table 1: This table gives the value of $h_{l} \sigma$ for each ring in the three different Bérenger layers used in the paper.

\begin{tabular}{||r|lll||}
\hline \hline Thickness & layer 1 & layer 2 & layer 3 \\
\hline \hline 6 & 1.03 & $0.92(0.54)$ & 1.03 \\
7 & 0.78 & 0.92 & 0.80 \\
8 & 0.74 & 0.92 & 0.80 \\
9 & $0.74(0.42)$ & $0.92(0.54)$ & $0.80(0.48)$ \\
\hline \hline
\end{tabular}

Table 2: This table shows the relative $L_{2}$ error (as a percentage) in the solution at $(x, y)=(-0.206,-0.0104)$ for various layers with various thicknesses. The structure of each layer is given in Table 1, and the thickness is the number of rings $\left(N_{\text {layer }}\right)$ added to the base mesh. The finite difference error is $0.18 \%$ (computed on a very large grid such that reflections from the outer boundary do not pollute the solution). Numbers in parenthesis are for $\Delta t=0.001$.

$\mathrm{RR} \mathrm{n}^{\circ} 3049$ 
results than the first order absorbing condition. These results indicate that it is worthwhile to test curvilinear Bérenger layers in three dimensional time harmonic computations which we are currently doing.

Much remains to be done. The theoretical analysis of the Bérenger system is incomplete. The presence (or absence hopefully) of exceptional frequencies needs to be investigated further, and an error estimate for the layer needs to be derived. Finally other discretization schemes (for example the curvilinear method in [24]) could be investigated to find the one best suited to the Bérenger layer. 


\section{References}

[1] W. Andrew, C. Balanis, And P. Tirkas, A comparison of the Berenger perfectly matched layer and the Lindman higher-order $A B C$ 's for the FDTD method, IEEE Microwave Guided Wave Lett., 5 (1995), pp. 192194.

[2] J. Berenger, A Perfectly Matched Layer for the Absorption of Electromagnetics Waves, J. Comput. Phys., 114 (1994), pp. 185-200.

[3] J.-P. BÉRenger, Perfectly matched layer for the FDTD solution of wave-structure interaction problems, IEEE Trans. Antennas Propagat., 44 (1996), pp. 110-117.

[4] M. Cai And H. Zhou, Proof of perfectly matched layer conditions in three dimensions, Electron. Lett., 31 (1995), pp. 1675-1676.

[5] B. Chen, D. G. Fang, And B. H. Zhou, Modified Berenger PML absorbing boundary condition for FD-TD meshes, IEEE Microwave Guided Wave Lett., 5 (1995), pp. 399-401.

[6] W. C. Chew And W. H. Weedon, A 3D perfectly matched medium from modified Maxwell's equations with stretched coordinates, Microwave Opt. Technol. Lett., 7 (1994), pp. 599-604.

[7] W. C. Chew, W. H. Weedon, And A. Sezginer, A 3-D perfectly matched medium by coordinate stretching and its absorption of static fields, in 11th Annual Review of Progress in Applied Computational Electromagnetics, vol. 1, Monterey, CA, Mar. 1995, pp. 482-489.

[8] F. Collino, Perfectly matched layer for the paraxial equation. J. Comp. Phys., submitted in 1995.

[9] D. Colton and R. Kress, Integral Equation Methods in Scattering Theory, John Wiley, New York, 1983.

[10] _ Inverse Acoustic and Electromagnetic Scattering Theory, SpringerVerlag, 1992.

RR $\mathrm{n}^{\circ} 3049$ 
[11] J. De Moerloose and M. A. Stuchly, Behavior of Berenger's ABC for evanescent waves, IEEE Microwave Guided Wave Lett., 5 (1995), pp. 344-346.

[12] C. Eswarappa And W. J. R. Hoefer, Implementation of Berenger absorbing boundary conditions in TLM by interfacing FDTD perfectly matched layers, Electron. Lett., 31 (1995), pp. 1264-1266.

[13] J. FAnG ANd Z. Wu, Generalized perfectly matched layer-An extension of Berenger's perfectly matched layer boundary condition, IEEE Microwave Guided Wave Lett, 5 (1995), pp. 451-453.

[14] D. GIVOLI, Non reflecting boundary conditions, Jour. Comp. Phys, 94 (1991).

[15] M. Gribbons, S.-K. Lee, and A. C. Cangellaris, Modification of Berenger's perfectly matched layer for the absorption of electromagnetics waves in layered media, in 11th Annual Review of Progress in Applied Computational Electromagnetics, vol. 1, Monterey, CA, Mar. 1995, pp. 498-503.

[16] R. HIGDON, Initial boundary value problems for hyperbolic systems, SIAM review, 28 (1977).

[17] D. Jones, The theory of electromagnetism, MacMillan, New York, 1964.

[18] D. S. Katz, E. T. Thiele, And A. Taflove, Validation and extension to three dimensions of the Berenger PML absorbing boundary condition for FD-TD meshes, IEEE Microwave Guided Wave Lett., 4 (1994), pp. 268270.

[19] A. Kirsch And P. Monk, An analysis of the coupling of finite element and Nyström methods in acoustic scattering, IMA J. on Numerical Analysis, 14 (1994), pp. 523-544.

[20] M.Israeli And S. Orszag, Approximation of radiation boundary conditions, Journal of Comp. Physics., 41 (1981), pp. 115-135. 
[21] R. Mittra And Ü. Pekel, A new look at the perfectly matched layer (PML) concept for the reflectionless absorption of electromagnetic waves, IEEE Microwave Guided Wave Lett., 5 (1995), pp. 84-86.

[22] P. Monk, A comparison of three mixed methods for the time dependent Maxwell equations, SIAM J. on Scientific and Statistical Computing, 13 (1992), pp. 1097-1122.

[23] C. M. Rappaport, Perfectly matched absorbing boundary conditions based on anisotropic lossy mapping of space, IEEE Microwave Guided Wave Lett., 5 (1995), pp. 90-92.

[24] A. Taflove, Computational Electrodynamics, Artech House, Boston, USA, 1995.

[25] Z. Wu AND J. FANG, Numerical implementation and performance of perfectly matched layer boundary condition for waveguide structures, IEEE Trans. Microwave Theory Tech., 43 (1995), pp. 2676-2683.

[26] — - Performance of the perfectly matched layer in modeling wave propagation in microwave and digital circuit interconnects, in 11th Annual Review of Progress in Applied Computational Electromagnetics, vol. 1, Monterey, CA, Mar. 1995, pp. 504-511.

$\mathrm{RR} \mathrm{n}^{\circ} 3049$ 


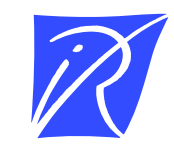

Unité de recherche INRIA Lorraine, Technopôle de Nancy-Brabois, Campus scientifique, 615 rue du Jardin Botanique, BP 101, 54600 VILLERS LÈS NANCY

Unité de recherche INRIA Rennes, Irisa, Campus universitaire de Beaulieu, 35042 RENNES Cedex

Unité de recherche INRIA Rhône-Alpes, 655, avenue de l' Europe, 38330 MONTBONNOT ST MARTIN

Unité de recherche INRIA Rocquencourt, Domaine de Voluceau, Rocquencourt, BP 105, 78153 LE CHESNAY Cedex

Unité de recherche INRIA Sophia-Antipolis, 2004 route des Lucioles, BP 93, 06902 SOPHIA-ANTIPOLIS Cedex

Éditeur

INRIA, Domaine de Voluceau, Rocquencourt, BP 105, 78153 LE CHESNAY Cedex (France)

ISSN 0249-6399 